OAK RIDGE NATIONAL LABORATORY

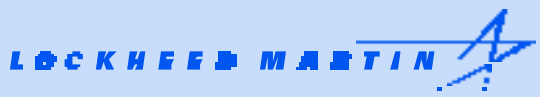

MANALEI MNDCPEFFTEDEY

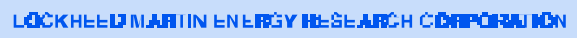
FAH THE UNITED STLTES IEPHFTMENT UF ENEREG

\section{CARTESIAN METHODS FOR THE SHALLOW WATER EQUATIONS ON A SPHERE}

J. B. Drake

P. N. Swarztrauber

D. L. Williamson 
This report has been reproduced directly from the best available copy.

Available to DOE and DOE contractors from the Office of Scientific and Technical Information, P.O. Box 62, Oak Ridge, TN 37831; prices available from (615) 576-8401.

Available to the public from the National Technical Information Service, U.S. Department of Commerce, 5285 Port Royal Rd., Springfield, VA 22161.

This report was prepared as an account of work sponsored by an agency of the United States Government. Neither the United States nor any agency thereof, nor any of their employees, makes any warranty, express or implied, or assumes any legal liability or responsibility for the accuracy, completeness, or usefulness of any information, apparatus, product, or process disclosed, or represents that its use would not infringe privately owned rights. Reference herein to any specific commercial product, process, or service by trade name, trademark, manufacturer, or otherwise, does not necessarily constitute or imply its endorsement, recommendation, or favoring by the United States Government or any agency thereof. The views and opinions of authors expressed herein do not necessarily state or reflect those of the United States Government or any agency thereof. 


\title{
CARTESIAN METHODS FOR THE SHALLOW WATER EQUATIONS ON A SPHERE
}

\author{
John B. Drake \\ Computer Science and Mathematics Division \\ Oak Ridge National Laboratory \\ Paul N. Swarztrauber and David L. Williamson \\ National Center for Atmospheric Research
}

Date Published: December 1999

\author{
Prepared by \\ OAK RIDGE NATIONAL LABORATORY \\ Oak Ridge, Tennessee 37831-6285 \\ managed by \\ LOCKHEED MARTIN ENERGY RESEARCH CORP. \\ for the \\ U.S. DEPARTMENT OF ENERGY \\ under contract DE-AC05-96OR22464
}




\section{Contents}

List of Figures $\quad$ iv

List of Tables $\quad$ v

Acknowledgments $\quad$ vi

Abstract $\quad$ vii

1 INTRODUCTION 1

2 THE SHALLOW WATER EQUATIONS ON A SPHERE 3

2.1 ROTATIONAL FORM OF THE MOMENTUM EQUATION . . . . . . . . . . . . 5

3 LOCAL CARTESIAN SPECTRAL APPROXIMATION

4 DISCRETE OPERATOR FORMULAS $\quad 8$

4.1 NUMERICAL RESULTS FOR THE GRADIENT APPROXIMATION $\ldots \ldots$

5 TEST CASES $\quad 11$

5.1 ADVECTION TEST . . . . . . . . . . . . . . . . . . 12

5.2 STEADY, ZONAL FLOW TEST . . . . . . . . . . . . . . . . . 12

5.3 NON-ANALYTIC, STEADY, ZONAL FLOW TEST . . . . . . . . . . . . . . 17

5.4 FORCED NONLINEAR SYSTEM WITH A TRANSLATING LOW . . . . . . . 18

5.5 ZONAL FLOW OVER AN ISOLATED MOUNTAIN . . . . . . . . . . . . 21

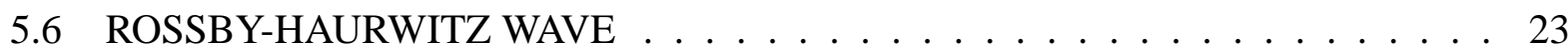

5.7 ANALYZED 500mb INITIAL CONDITIONS . . . . . . . . . . . . . . . . . 27

6 CONCLUSIONS $\quad 29$

A SPHERICAL HARMONIC POLYNOMIALS 1 


\section{List of Figures}

1 Approximation stencil for a regular point of an icosahedral grid. . . . . . . . . . 11

2 Convergence of the Gradient Approximations. . . . . . . . . . . . . . . . . 12

3 Relative RMS Error in height; Test Case $1 ; q=1,2,3,4 \ldots \ldots$. . . . . . . . . . 13

4 Relative RMS Error in velocity; Test Case 2; $q=2,3,4$; Standard Form. . . . . . . 14

5 Relative RMS Error in height; Test Case 2; $q=2,3,4$; Standard Form. . . . . . . . 14

6 Relative RMS Error in Velocity; Test Case 2; $q=2,3,4$; Rotational Form. . . . . . 15

$7 \quad$ Relative RMS Error in Height; Test Case 2; $q=2,3,4$; Rotational Form. . . . . . . 15

8 Error in Geopotential at 5 days; Test Case 2; $q=4$; Rotational Form. . . . . . . . . 16

9 Comparison of geopotential Fourier spectrum for Case 2; $q=3$; Rotational Form. . 17

10 Test Case 2, Perturbed Spectral Geopotential Error (a) at Day 4, (b) at Day 5. . . . 18

11 Relative RMS Error in Velocity; Test Case 3; $q=2,3,4$; Standard Form. . . . . . . 19

12 Relative RMS Error in Height; Test Case 3; $q=2,3,4$; Standard Form. . . . . . . . 19

13 Relative RMS Error in Velocity; Test Case 3; $q=2,3,4$; Rotational Form. . . . . . 20

14 Relative RMS Error in Height; Test Case 3; $q=2,3,4$; Rotational Form. . . . . . . 20

15 Error in Geopotential at 5 days; Test Case $3 ; q=4$; Rotational Form. . . . . . . . . 21

16 Relative RMS Error in Velocity; Test Case 4; $q=2,3,4$; Rotational Form. . . . . . 22

17 Relative RMS Error in Height; Test Case 4; $q=2,3,4$; Rotational Form. . . . . . . 22

18 Conserved integral quantities; Test Case 5; $q=4$; Rotational Form. . . . . . . . . . 23

19 Geopotential at 15 days; Test Case 5; $q=4$; Rotational Form. . . . . . . . . . . . . . 24

20 Conserved integral quantities; Test Case 6; $q=4$; Rotational Form. . . . . . . . . . 24

21 Geopotential at 14 days; Test Case $6 ; q=4$; Rotational Form. . . . . . . . . . . . . 25

22 Cartesian Geopotential at Day 1. . . . . . . . . . . . . . . . . . . 26

23 Comparison of geopotential Fourier spectrum for Case $6 ; q=3$; Rotational Form. . 26

24 Test Case 6, (a) Cartesian Geopotential at Day 5, (b) Perturbed Spectral Geopoten-

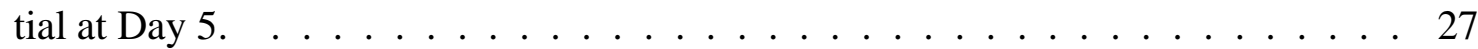

25 Conserved integral quantities; Test Case 7a; $q=4$; Rotational Form. . . . . . . . . 28

26 Test Case 7a, (a) Reference Solution at Day 1, (b) Cartesian Geopotential at Day 1. 28

27 Test Case 7, (a) Reference Solution at 5 Day, (b) Cartesian Geopotential at 5 Day. . 29

28 Test Case 7b, (a) Reference Solution at Day 1, (b) Cartesian Geopotential at Day 1. 30

29 Test Case 7, (a) Reference Solution at 5 Day, (b) Cartesian Geopotential at 5 Day. . 30

30 Conserved integral quantities. Test Case $7 \mathrm{~b}, q=4$; Rotational Form. . . . . . . . . 31

31 Test Case 7c, (a) Reference Solution at Day 1, (b) Cartesian Geopotential at Day 1. 31

32 Test Case 7c, (a) Reference Solution at 5 Day, (b) Cartesian Geopotential at 5 Day. 32

33 Conserved integral quantities. Test Case 7c, $q=4$; Rotational Form. . . . . . . . 32 


\section{List of Tables}

1 Geometric information for icosahedral grids. . . . . . . . . . . . . . 10 


\section{Acknowledgements}

This report is one in a series of documents describing the the development of numerical methods for global climate modeling. The work reported is sponsored by the CHAMMP program of the Department of Energy's Office of Biological and Environmental Research, Environmental Sciences Division. We gratefully acknowledge the support of the CHAMMP program and the National Science Foundation who jointly support the work at the National Center for Atmospheric Research in Boulder. 


\begin{abstract}
The shallow water equations in a spherical geometry are solved using a 3-dimensional Cartesian method. Spatial discretization of the 2-dimensional, horizontal differential operators is based on the Cartesian form of the spherical harmonics and an icosahedral (spherical) grid. Computational velocities are expressed in Cartesian coordinates so that a problem with a singularity at the pole is avoided. Solution of auxiliary elliptic equations is also not necessary. A comparison is made between the standard form of the Cartesian equations and a rotational form using a standard set of test problems. Error measures and conservation properties of the method are reported for the test problems.
\end{abstract}




\section{INTRODUCTION}

This report is one in a series of documents describing the development of numerical methods for global climate modeling. The work reported is sponsored by the CHAMMP program of the Department of Energy's Office of Biological and Environmental Research. CHAMMP is an acronym for Computer Hardware, Advanced Mathematics and Model Physics. Its goal is the development of advanced climate models with considerably improved throughput, accuracy and realism over existing models.

The use of triangular meshes for the solution of partial differential equations (PDE's) on a spherical domain is attractive for two reasons. Triangles allow nearly uniform meshes while rectangular meshes suffer the problem of varying resolution near the poles. Secondly, triangles require only a simple data structure for use with adaptive mesh techniques or for meshes that resolve irregular features. Adapting a mesh to fit a coastline is an obvious example.

Several early papers investigated the use of icosahedral- triangular meshes $[12,13,19,20,21$, 22]. The barotropic vorticity equation and the shallow water equations on the sphere served as the primary equation sets for testing the numerical methods because of their relevance in atmospheric flow models. A review article [23] gives further references to that early work.

More recently an icosahedral method based on the stream function and velocity potential formulation of the shallow water equations with a control volume discretization has been proposed by Masuda [9]. The method was refined and tested on a standard set of cases [24] by Heikes and Randall in [5, 6]. Other icosahedral methods have also been proposed in [2].

Renewed interest in these methods springs from advances in computing and numerical analysis. The introduction of massively parallel computers has prompted the reexamination of many classical algorithms. The granularity of tasks that can be performed in parallel appears to be finer for the finite difference and finite element methods than for spectral transform methods which dominate global atmospheric modeling. This offers the possibility for the effective use of many small processors of a parallel computer.

The Cartesian form of the shallow water equations was proposed by Swarztrauber in [24] and further developed in [17]. This alternative formulation avoids the usual singularity in the velocity at the pole by expressing velocities in a three dimensional Cartesian form instead of in spherical coordinates. The introduction of three dimensional velocities necessitates a change of the form of the shallow water equations. At first sight, this form appears more complicated and probably more 
expensive computationally. However, a closer examination shows the Cartesian formulation to be compact and computationally simple.

The pole problem can also be partially addressed by introducing new scalar variables derived from the velocity. The stream function/velocity potential or the vorticity/divergence function are the usual choices. The resulting system of equations then involves elliptic equations relating the new scalar variables. Since there is no introduction of new scalar variables in the Cartesian formulation, the introduction of elliptic equations is avoided. Thus, the Cartesian method has a significantly lower operation count than those methods requiring the solution of an elliptic equation.

The Cartesian geometry of the sphere and the discretization of the sphere using the points of an icosahedral triangular mesh also lead to a computational economy at the poles. The distances between points of this mesh are nearly uniform, thus, there is not an artificially severe CFL restriction on timestep arising from a longitudinal concentration of points near the pole. There is no need to filter the solution near the poles, a step that can be costly for some methods and can introduce errors on all scales.

The Cartesian formulation was used with the calculation of derivatives using a spectral vector harmonic method in previous research [16]. In this paper, we consider the Cartesian formulation with the calculation of derivatives using a stencil of points located on an icosahedral grid. The derivative approximations might be characterized as locally spectral in that they are based on spherical harmonics, but only use a local stencil of points like a finite difference method. We show, by numerical experiments, that the method of approximating differential operators on the icosahedral mesh is accurate and converges as the mesh is refined.

The discretization is then applied to the shallow water equations on a sphere and tested extensively on a set of standard cases for shallow water equation solvers. These tests highlight many of the positive properties of the method as well as expose some of its shortcomings. We pay particular attention to the conservation properties of the computed solutions. By changing the formulation of the shallow water equations to a "rotational form," the conservation and energy stability is significantly improved. 


\section{THE SHALLOW WATER EQUATIONS ON A SPHERE}

The momentum and mass continuity equations for shallow water flows can be written in advective form:

$$
\frac{d \mathbf{v}}{d t}=-f \mathbf{k} \times \mathbf{v}-g \nabla h+\mathbf{F}_{\mathbf{v}}
$$

and

$$
\frac{d h^{*}}{d t}=-h^{*} \nabla \cdot \mathbf{v}+F_{h}
$$

where the substantial derivative is given by

$$
\frac{d}{d t}() \equiv \frac{\partial}{\partial t}()+\mathbf{v} \cdot \nabla()
$$

The velocity is referred to a rotating Cartesian frame and the components of $\mathbf{v}=(u, v)$ are in the longitudinal and latitudinal directions, respectively. The height of the free surface is defined $h=h^{*}+h_{s}$, where $h^{*}$ is the depth of the fluid and the bottom surface height is given by the time invariant function $h_{s}$. External forcing, if present, is included in $\mathbf{F}_{\mathbf{v}}=\left(F_{u}, F_{v}\right)$ and $F_{h}$. This equation is not in conservative form and, consequently, the numerical methods we develop will not be strictly conservative.

It may be advantageous to evaluate the horizontal (surface) derivatives using a Cartesian form. This form was developed in detail in previous research [17]. By extending the surface vector $\mathbf{v}=(u, v)^{T}$ to the three-dimensional $\mathbf{v}_{s}=(w, v, u)^{T}$, the momentum equations can be embedded in the system

$$
\frac{\partial \mathbf{v}_{s}}{\partial t}+\mathbf{S}\left(\mathbf{v}_{S}\right) \mathbf{v}_{s}+\boldsymbol{\alpha}+\boldsymbol{\beta}+\boldsymbol{\gamma}+\boldsymbol{\delta}=0
$$

where

$$
\mathbf{S}\left(\mathbf{v}_{s}\right)=\left(\begin{array}{ccc}
\frac{\partial w}{\partial r} & \frac{1}{a}\left(\frac{\partial w}{\partial \theta}-v\right) & \frac{1}{a \cos \theta}\left(\frac{\partial w}{\partial \lambda}-u \cos \theta\right) \\
\frac{\partial v}{\partial r} & \frac{1}{a}\left(\frac{\partial v}{\partial \theta}+w\right) & \frac{1}{a \cos \theta}\left(\frac{\partial v}{\partial \lambda}-u \sin \theta\right) \\
\frac{\partial u}{\partial r} & \frac{1}{a} \frac{\partial u}{\partial \theta} & \frac{1}{a \cos \theta}\left(\frac{\partial u}{\partial \lambda}-v \sin \theta+w \cos \theta\right)
\end{array}\right)
$$

$\lambda$ is the longitude coordinate, $\theta$ is the latitude coordinate, $r$ is the radial coordinate ( $r=a$ at the 
earth's surface) and

$$
\begin{aligned}
& \boldsymbol{\alpha}=\left(\begin{array}{c}
\frac{u^{2}+v^{2}}{a} \\
0 \\
0
\end{array}\right), \\
& \boldsymbol{\beta}=\left(\begin{array}{c}
0 \\
\frac{g}{a} \frac{\partial h}{\partial \theta} \\
\frac{g}{a \cos \theta} \frac{\partial h}{\partial \lambda}
\end{array}\right), \\
& 0 \\
& \boldsymbol{\gamma}=\left(\begin{array}{c}
-F_{v} \\
-F_{u}
\end{array}\right),
\end{aligned}
$$

and

$$
\boldsymbol{\delta}=\left(\begin{array}{c}
0 \\
f u \\
-f v
\end{array}\right)
$$

If we define $\mathbf{V}=(X, Y, Z)^{T}$ as the velocity in Cartesian coordinates $(x, y, z)$ then

$$
\mathbf{v}_{s}=\mathbf{Q V}
$$

where

$$
\mathbf{Q}=\left(\begin{array}{ccc}
\cos \theta \cos \lambda & \cos \theta \sin \lambda & \sin \theta \\
-\sin \theta \cos \lambda & -\sin \theta \sin \lambda & \cos \theta \\
-\sin \lambda & \cos \lambda & 0
\end{array}\right)
$$

Substituting Equation 10 into Equation 4 and multiplying by $\mathbf{Q}^{T}$ we obtain the Cartesian form

$$
\frac{\partial \mathbf{V}}{\partial t}+\mathbf{C V}+\mathbf{Q}^{T}(\boldsymbol{\alpha}+\boldsymbol{\beta}+\boldsymbol{\gamma}+\boldsymbol{\delta})=0
$$

In this equation,

$$
\mathbf{C}=\mathbf{Q}^{T} \mathbf{S Q}=\left(\begin{array}{ccc}
\frac{\partial X}{\partial x} & \frac{\partial X}{\partial y} & \frac{\partial X}{\partial z} \\
\frac{\partial Y}{\partial x} & \frac{\partial Y}{\partial y} & \frac{\partial Y}{\partial z} \\
\frac{\partial Z}{\partial x} & \frac{\partial Z}{\partial y} & \frac{\partial Z}{\partial z}
\end{array}\right)
$$




$$
\begin{gathered}
\mathbf{Q}^{T} \boldsymbol{\alpha}=\frac{1}{a^{2}}\left(\begin{array}{c}
x\left(X^{2}+Y^{2}+Z^{2}\right) \\
y\left(X^{2}+Y^{2}+Z^{2}\right) \\
z\left(X^{2}+Y^{2}+Z^{2}\right)
\end{array}\right), \\
\mathbf{Q}^{T} \boldsymbol{\delta}=\frac{2 \Omega z}{a^{2}}\left(\begin{array}{ccc}
0 & -z & y \\
z & 0 & -x \\
-y & x & 0
\end{array}\right)\left(\begin{array}{c}
X \\
Y \\
Z
\end{array}\right),
\end{gathered}
$$

and

$$
\mathbf{Q}^{T} \boldsymbol{\beta}=g \mathbf{P} \nabla_{c} h
$$

where

$$
\mathbf{P}=\frac{1}{a^{2}}\left(\begin{array}{ccc}
a^{2}-x^{2} & -x y & -x z \\
-x y & a^{2}-y^{2} & -y z \\
-x z & -y z & a^{2}-z^{2}
\end{array}\right)
$$

and

$$
\nabla_{c} h=\left(\frac{\partial h}{\partial x}, \frac{\partial h}{\partial y}, \frac{\partial h}{\partial z}\right)^{T} .
$$

Similarly, the continuity equation in Cartesian form is

$$
\frac{\partial h^{*}}{\partial t}+\mathbf{V}^{T} \mathbf{P} \nabla_{c} h^{*}+h^{*} \nabla_{c} \cdot \mathbf{V}=F_{h} .
$$

The matrix $P$ projects an arbitrary Cartesian vector onto a plane that is tangent to the sphere at the point $(x, y, z)$.

\subsection{ROTATIONAL FORM OF THE MOMENTUM EQUATION}

The vorticity, $\zeta$, is defined in the spherical coordinate system as $\zeta \equiv \mathbf{k} \cdot \nabla \times \mathbf{v}$. Using the vector identity

$$
\mathbf{v} \cdot \nabla \mathbf{v}=\nabla\left(\frac{\mathbf{v} \cdot \mathbf{v}}{2}\right)+\zeta \mathbf{k} \times \mathbf{v},
$$

the momentum equation can be written as

$$
\frac{\partial \mathbf{v}}{\partial t}=-(\zeta+f) \mathbf{k} \times \mathbf{v}-\nabla\left(g h+\frac{\mathbf{v} \cdot \mathbf{v}}{2}\right)+\mathbf{F}_{\mathbf{v}} .
$$


Changing variables to Cartesian velocities the resulting Cartesian equation is

$$
\frac{\partial \mathbf{V}}{\partial t}+\mathbf{Q}^{T}(\boldsymbol{\Lambda}+\boldsymbol{\gamma}+\boldsymbol{\Delta})=0
$$

In this equation,

$$
\mathbf{Q}^{T} \boldsymbol{\Delta}=\frac{(\zeta+2 \Omega z)}{a^{2}}\left(\begin{array}{ccc}
0 & -z & y \\
z & 0 & -x \\
-y & x & 0
\end{array}\right)\left(\begin{array}{c}
X \\
Y \\
Z
\end{array}\right)
$$

and

$$
\mathbf{Q}^{T} \boldsymbol{\Lambda}=\mathbf{P} \nabla_{c}\left(g h+\frac{\mathbf{V} \cdot \mathbf{V}}{2}\right)
$$

Since the curl is invariant under coordinate transformation, we have $\zeta \equiv \mathbf{k} \cdot \nabla_{c} \times \mathbf{V}$ where $\mathbf{k}$ is the unit vector in the direction normal to the sphere at the point $(x, y, z)$. That is, $\mathbf{k}=\frac{\mathbf{x}}{a}$, where the notation in Cartesian coordinates should not be confused with the standard notation $\mathbf{k}$ for the unit vector in the $z$-direction. The Cartesian curl is the standard

$$
\nabla_{c} \times \mathbf{V}=\left(\begin{array}{c}
\frac{\partial Z}{\partial y}-\frac{\partial Y}{\partial z} \\
\frac{\partial X}{\partial z}-\frac{\partial Z}{\partial x} \\
\frac{\partial Y}{\partial x}-\frac{\partial X}{\partial y}
\end{array}\right)
$$

These derivatives are available from the $\mathbf{C}$ matrix described above.

The rotational form of the momentum equation has one excellent property for collocation methods[1]: it is semi-energy conserving. This can be seen by considering the discrete kinetic energy equation obtained by the vector multiplication of the momentum equation with the velocity. Ignoring forcing terms and surface orography:

$$
\mathbf{V} \cdot \frac{\partial \mathbf{V}}{\partial t}=-\frac{(\zeta+2 \Omega z)}{a^{2}} \mathbf{V} \cdot\left(\begin{array}{ccc}
0 & -z & y \\
z & 0 & -x \\
-y & x & 0
\end{array}\right)\left(\begin{array}{c}
X \\
Y \\
Z
\end{array}\right)-\mathbf{V} \cdot \mathbf{P} \nabla_{c}\left(g h+\frac{\mathbf{V} \cdot \mathbf{V}}{2}\right)
$$

The troublesome advection term is split into two parts, one purely normal to the velocity and the other buried in the gradient as the kinetic energy. The first term vanishes identically when 
multiplied by the Cartesian velocity because the velocity is tangent to the surface of the sphere:

$$
\frac{\partial \frac{\mathbf{V} \cdot \mathbf{V}}{2}}{\partial t}=-\mathbf{V} \cdot \mathbf{P} \nabla_{c}\left(g h+\frac{\mathbf{V} \cdot \mathbf{V}}{2}\right)
$$

Defining the total energy by $E=g h+\frac{\mathbf{V} \cdot \mathbf{V}}{2}$ and adding Equation 27 to Equation 19, the discrete energy equation is

$$
\frac{\partial E}{\partial t}=-\mathbf{V} \cdot \mathbf{P} \nabla_{c}(E)-\mathbf{V} \cdot \mathbf{P} \nabla_{c} g h-g h \nabla_{c} \cdot \mathbf{V}
$$

For energy stability, the integral of the discrete energy must be constant:

$$
0=\frac{\partial \bar{E}}{\partial t}
$$

where, by way of notation, the overbar is the integral over the sphere, $\overline{()}=\int_{S^{2}}()$. Integrating Equation 28 yields

$$
0=-\overline{\left(\mathbf{V} \cdot \mathbf{P} \nabla_{c}(E)\right)}-\overline{\left(\mathbf{V} \cdot \mathbf{P} \nabla_{c} g h+g h \nabla_{c} \cdot \mathbf{V}\right)}
$$

If the discrete gradient is the negative adjoint of the discrete divergence, the energy equation becomes

$$
0=+\overline{\left(E \nabla_{c} \cdot \mathbf{V}\right)}-\overline{\left(\nabla_{c} \cdot(g h \mathbf{V})\right)}
$$

For a divergence free velocity, the first term vanishes, and the second term is the condition of global conservation of mass. Therefore, the discrete energy will be conserved if these conditions are met. Using the rotational form in collocation methods improves the nonlinear energy stability of the numerical method and reduces the need for artificial diffusion to enhance stability.

\section{LOCAL CARTESIAN SPECTRAL APPROXIMATION}

The spherical harmonic functions form a basis for functions defined on the surface of the sphere. They have long been used in climate and weather models as the basis for the spectral method [8] and for the approximation of derivatives on the surface of the sphere [15]. The spherical harmonic, $Y_{n}^{m}$, can be defined with the normalized associated Legendre functions, $P_{n}^{m}(\theta)$, by

$$
Y_{n}^{m}(\lambda, \theta)=e^{i m \lambda} \bar{P}_{n}^{m}(\theta)
$$


The normalized associated Legendre polynomials can be defined from Rodrigues' formula [14]:

$$
\bar{P}_{n}^{m}(\theta)=(-1)^{m}\left[\frac{2 n+1}{2} \frac{(n-m) !}{(n+m) !}\right]^{1 / 2} \frac{1}{2^{n} n !}(\sin \theta)^{m} \frac{d^{m+n}}{d z^{m+n}}\left(z^{2}-1\right)^{n}
$$

where $z=\cos \theta$ and $\theta$ is colatitude. (In this section only, $\theta$ refers to colatitude while in other sections it refers to latitude.) Equations 32 and 33 are combined to give a formula for the Cartesian representation of the spherical harmonics [18]:

$$
Y_{n}^{m}(x, y, z)=C_{n}^{m}(x+i y)^{m} \frac{d^{m+n}}{d z^{m+n}}\left(z^{2}-1\right)^{n}
$$

where

$$
C_{n}^{m}=\frac{(-1)^{m}}{2^{n} n !}\left[\frac{2 n+1}{2} \frac{(n-m) !}{(n+m) !}\right]^{1 / 2} .
$$

Each spatial field could be approximated in Cartesian coordinates by a series of trivariate polynomials. For example,

$$
\phi(x, y, z)=\sum_{m, n} c_{n}^{m} Y_{n}^{m}(x, y, z)
$$

where the $c_{n}^{m}$ are coefficients of the trivariate polynomials $Y_{n}^{m}$. To find the $c_{n}^{m}$ 's in the expression for $\phi$, a least squares problem could be solved to fit $\phi$ data at a set of points on the surface of the sphere with the expansion. Depending on how many points this involves and how many terms are taken in the spectral expansion, this is either a full rank or a rank deficient least squares problem. For example, on an icosahedral grid each grid point has six or seven nearest neighbors. Using a second order interpolant, we have nine spherical harmonics. This would be a local spectral approximation.

\section{DISCRETE OPERATOR FORMULAS}

As an alternative to interpolating a field and then differentiating the formula, we can approximate the differential operators directly by requiring that the discrete operators act correctly on the selected basis functions. Given a cluster of points $\left\{p_{l}\right\}, l=0, \ldots, n p-1$ on the surface of the sphere and a tabulation of a function $U,\left\{U\left(p_{l}\right)\right\}$ about the point $p_{0}$, we wish to determine coefficients $c_{l}$ such that

$$
L(U)\left(p_{0}\right) \approx \sum_{l=0}^{n p-1} c_{l} U\left(p_{l}\right)
$$


The sense of the approximation $[\approx]$ must be described. We require Equation 37 to hold for all spherical harmonics through some number $N$ :

$$
L\left(r^{n} Y_{n}^{m}\right)\left(p_{0}\right) \cong \sum_{l=0}^{n p-1} c_{l} r^{n} Y_{n}^{m}\left(p_{l}\right)
$$

The spherical harmonics $Y_{n}^{m}$ are ordered so that with increasing number the degree increases (see Appendix A for a listing of the harmonics as trivariate polynomials). This system is then solved for the $c_{l}$. A different set of $c_{l}$ is required for each point $p_{0}$ and the stencil of points around it. For the shallow water equations, the stencil coefficients are calculated for each of the linear operators, $L(U)=\frac{\partial U}{\partial x}, \frac{\partial U}{\partial y}, \frac{\partial U}{\partial z}$, and the Laplacian, $\Delta U$. This approach is general and is applicable to any distribution of points on the sphere.

The sense of the approximation in Equation 37 is as a least squares problem for Equation 38. The problem can be stated in matrix form: find $\mathbf{c}$ which minimizes

$$
\|\mathbf{H c}-\mathbf{d}\|_{2}^{2}
$$

where $\mathbf{H}$ is a $N \times n p$ matrix of spherical harmonics evaluated at points of the stencil and $\mathbf{d}$ is the vector of the exact linear operator applied to the harmonics evaluated at the point. The least squares problem can be solved elegantly using the singular value decomposition (SVD) [7]. Let $\mathbf{H}=\mathbf{U S V}^{T}$ be the SVD. The matrix $\mathbf{S}=\operatorname{diag}\left(\sigma_{1}, \sigma_{2}, \ldots, \sigma_{n p}\right)$ is the matrix of singular values in descending order. The solution to the least squares problem is

$$
\mathbf{c}=\mathbf{V} \mathbf{S}^{-\mathbf{1}} \mathbf{U}^{T} \mathbf{d}
$$

The choice of $N$ and $n p$ determine the formal accuracy and smoothness of the derivative approximations. In general, we choose $n p$ points that are nearly symmetric about the point $p_{0}$. The number of points used in the stencil will determine the efficiency of the method because evaluation of the derivatives requires a combination of values from these neighboring points. For the icosahedral grid, each point has five or six immediate neighbors $(n p=7)$ (See Fig. 1). If the immediate neighbors of each of these points are included in the stencil, then $n p=19$.

A smoothing parameter that can be introduced in the approximation is the truncation level in the SVD solution. The diagonal matrix of singular values, $\mathbf{S}$, can be used to smooth the least squares 


\begin{tabular}{||lllllll||}
\hline $\mathrm{q}$ & Grid Points & Triangles & $h_{\min }(\mathrm{km})$ & $h_{\max }(\mathrm{km})$ & $h_{\text {ave }}(\mathrm{km})$ & $h_{\min } / h_{\max }$ \\
\hline- & 12 & 20 & 6699.0 & 6699.0 & 6699.0 & 1.0000 \\
0 & 42 & 80 & 3482.0 & 3938.0 & 3710.0 & 0.8843 \\
1 & 162 & 320 & 1613.0 & 2070.0 & 1901.0 & 0.7792 \\
2 & 642 & 1280 & 761.1 & 1049.0 & 956.2 & 0.7255 \\
3 & 2562 & 5120 & 368.4 & 526.3 & 478.8 & 0.7001 \\
4 & 10242 & 20480 & 181.2 & 263.4 & 239.5 & 0.6878 \\
\hline
\end{tabular}

Table 1: Geometric information for icosahedral grids.

solution when the system is underdetermined. By truncating the singular values that are smaller than some variable tolerance, a solution for $\mathbf{c}$ is obtained which supresses unwanted oscillations. This truncation gives a solution regardless of the underdetermined or overdetermined nature of the least squares problem. We have found it advantageous to use the same truncation at all points. For $n p=7$, we truncate at six because the primary points of the icosahedron have only five neighbors. The coefficients are unique at these points and of minimum norm at the other points.

\subsection{NUMERICAL RESULTS FOR THE GRADIENT APPROXIMATION}

The basic icosahedral mesh consists of 20 spherical triangles on 12 grid points. Each of the twelve points of the mesh is connected to five neighboring points. The refinements of this mesh are subdivisions of the twenty base triangles. By placing three points on each edge of each large triangle, and dividing each triangle into three subtriangles, the $q=0$ mesh is obtained by projection of the points (and edges) onto the surface of the sphere. Halving this mesh results in the $q=1$ mesh and halving again gives the $q=2$ mesh. The number of points in the mesh is given by the formula,

$$
G P(q)=5\left(2^{2 q+3}\right)+2
$$

Table 1 gives geometric information about the different icosahedral meshes.

To check the accuracy of the gradient approximations, a test function exhibiting all modes was used:

$$
\phi(\lambda, \theta)=a(\exp x+\exp y+\exp z) .
$$

The errors in the following tables are derived from the Cartesian approximation of the gradient of this function. The exact values of the function at the vertices of the icosahedral mesh are computed and used in the difference formulas to approximate the Cartesian derivatives. The Cartesian gra- 
dient is then transformed to spherical coordinates and compared with the exact gradient of $\phi$. The error reported is the $l^{2}$-error over the points of the icosahedral mesh.

The choice of $N$, the number of spherical harmonics and $n p$, the number of points in the stencil, determines the formal accuracy of the method. If $N=9$, then spherical harmonics of second order are used. If $N=16$, then third order harmonics are included. For $N=25$, fourth order harmonics are included. On an icosahedral grid each point is in a cluster of six or seven. Adding neighbors of these points with some symmetry leads to either thirteen or nineteen point clusters. These clusters define the stencil of the discrete operator (see Figure 1 ).

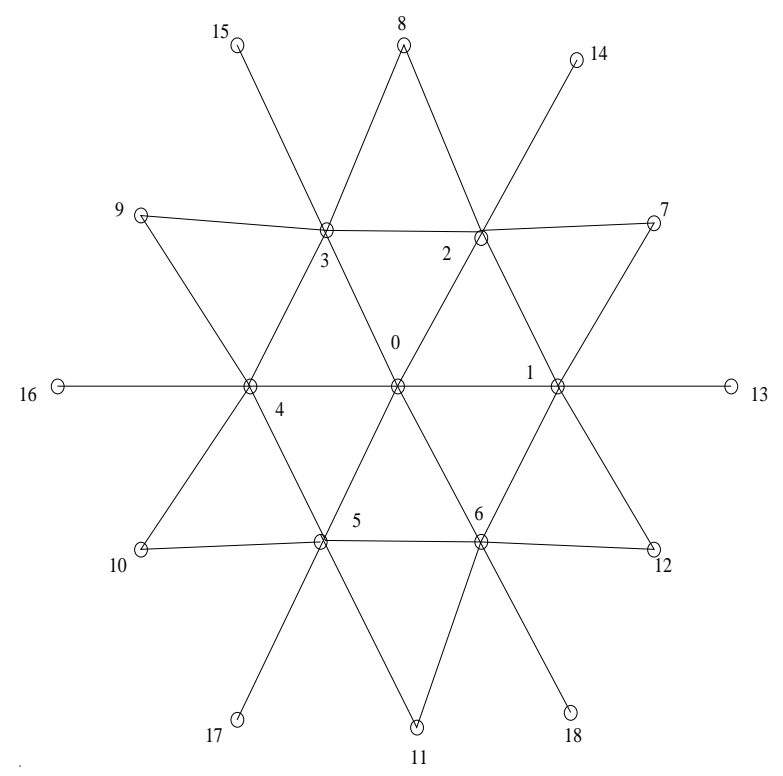

Fig. 1: Approximation stencil for a regular point of an icosahedral grid.

Plotting the error in Figure 2 with a log-log scale shows the second order (or greater) convergence of the approximation as the mesh spacing is decreased. Though the gradient approximations appear to be accurate for higher order spherical harmonics, we will restrict attention in this paper to the quadratic case using $N=9$ and $n p=7$.

\section{TEST CASES}

A set of test cases for the shallow water equations on a sphere are detailed in [24]. These cases provide a rigorous test for methods as well as allowing for comparison between methods. 


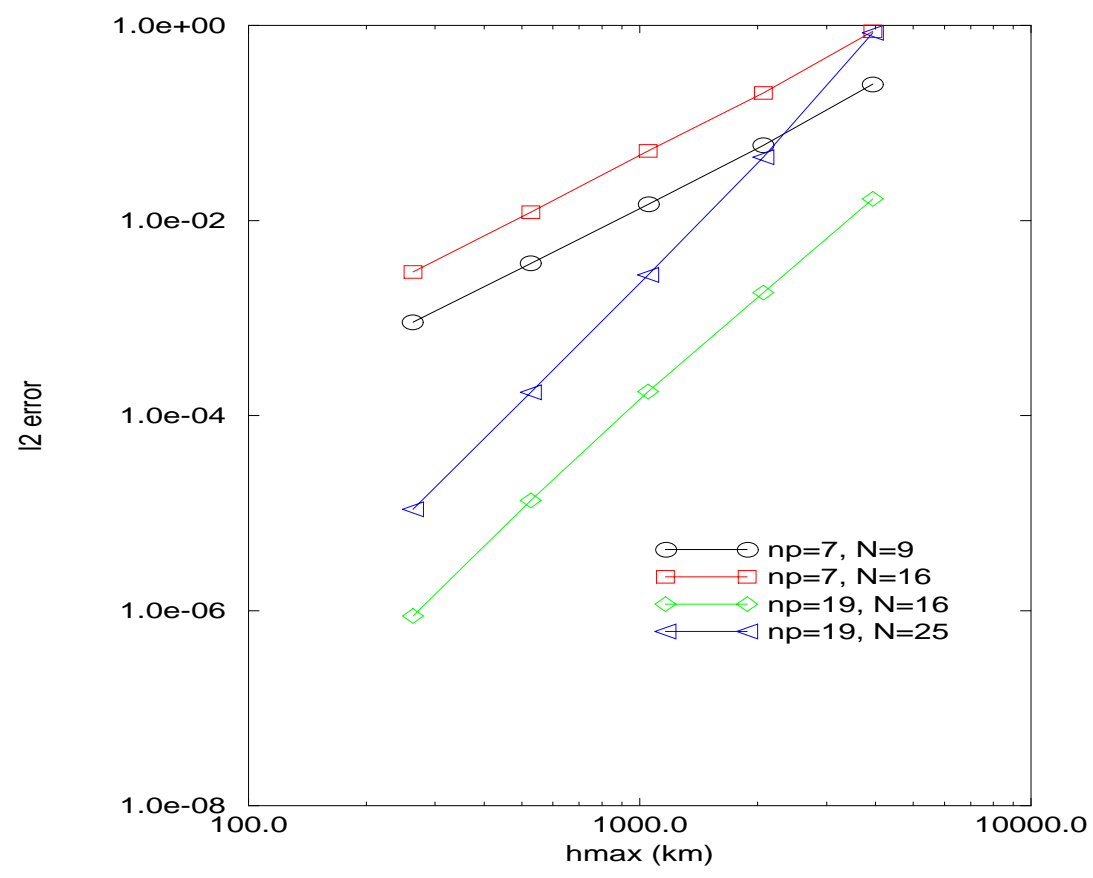

Fig. 2: Convergence of the Gradient Approximations.

\subsection{ADVECTION TEST}

Test Case 1 is a pure advection problem in which a cosine bell is blown around the sphere under a constant velocity field. Figure 3 shows the relative RMS error in the geopotential field as a function of time for a variety of grids. The operators are approximated with quadratic spherical harmonics and use only nearest neighbors. No diffusion was used for this case. The timestep was 1200 seconds for meshes $q=1,2,3$ and 600 seconds for the $q=4$ mesh. As can be seen from Figure 3, the error decreases with mesh refinement. A contour plot of the error (not shown) reveals a significant wake behind the cosine bell as a result of the centered spatial approximation. Thus, while convergent, the method has deficiencies as an advection scheme.

\subsection{STEADY, ZONAL FLOW TEST}

Test Case 2 is a steady, non-linear zonal flow rotated through an angle, $\alpha=\frac{\pi}{4}$. It tests the ability of the code to maintain a steady state solution independent of the grid orientation and gives a good idea of the accuracy of the methods. The velocity and geopotential for this test case are exactly representable with the spherical harmonics of second order. Hence, the local spherical harmonic approximations for the derivative operators are able to capture the steady state solution extremely well. Figures 4 and 5 show the error, as a function of time, in the velocity (using the relative 


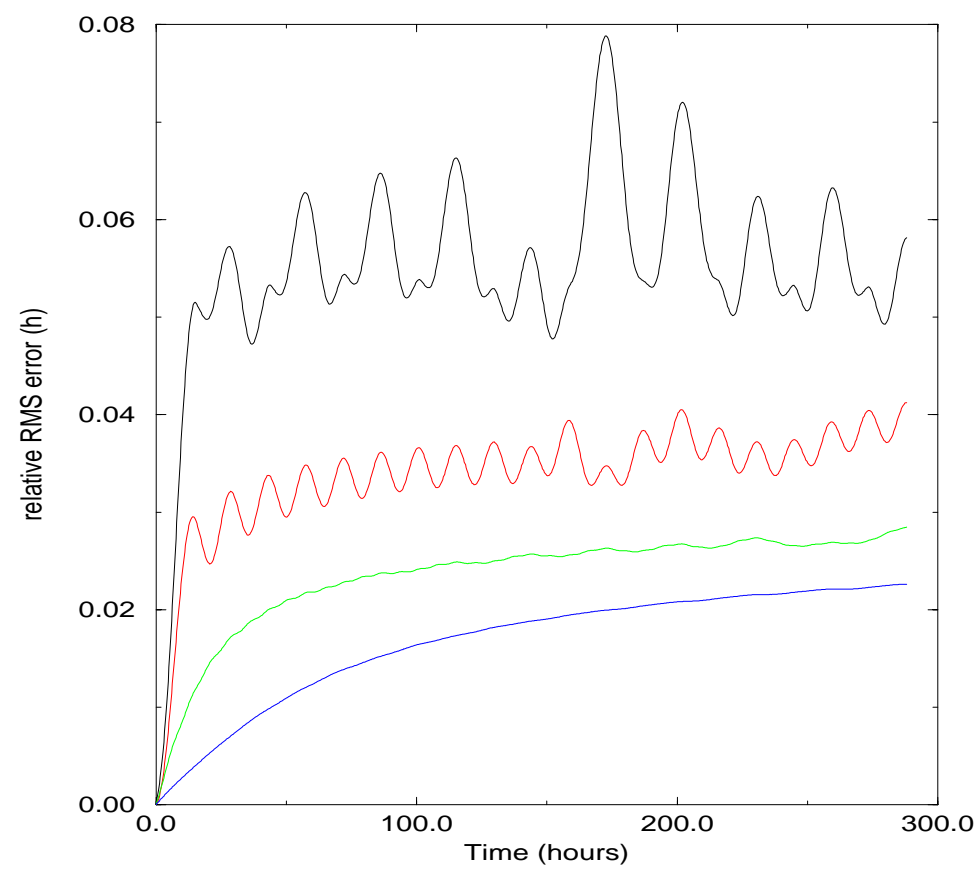

Fig. 3: Relative RMS Error in height; Test Case 1; $q=1,2,3,4$.

RMS error with the exact steady solution) and the RMS error in the height field, respectively. The $q=2,3$ integrations used a time step of 1200 seconds for the 5 day (120 hour) simulations while the $q=4$ mesh used a 600 second timestep. For the standard formulation, two diffusion terms were added. The momentum equation was modified with a diffusion operator, $\varepsilon_{V} \Delta V$, where $\varepsilon_{V}=8000$. Similarly, the height equation was modified with a diffusion coefficient of $\varepsilon_{h}=20000$. For the rotational formulation, no diffusion was added.

The rotational form gives somewhat better results with no diffusion added. Figures 6 and 7 show the velocity error and the height error for the rotational form of the Cartesian equations also using the quadratic approximation on six or seven neighbors. The error growth for this form is more controlled.

A contour plot of the absolute geopotential error is given in Figure 8. The error is measured at five days. Clearly evident are the base points of the icosahedral grid where the difference stencil involves six rather than seven points.

The error is held to a small level. However, the error shown in Figure 8 exhibits a curious wave \#5 mode. Other methods based on latitude-longitude grids do not exhibit a systematic error in wave \#5. Since there are five icosahedral points around the earth at a given latitude, this raises questions about the influence of the truncation error on the computed solution. What is the expected growth 


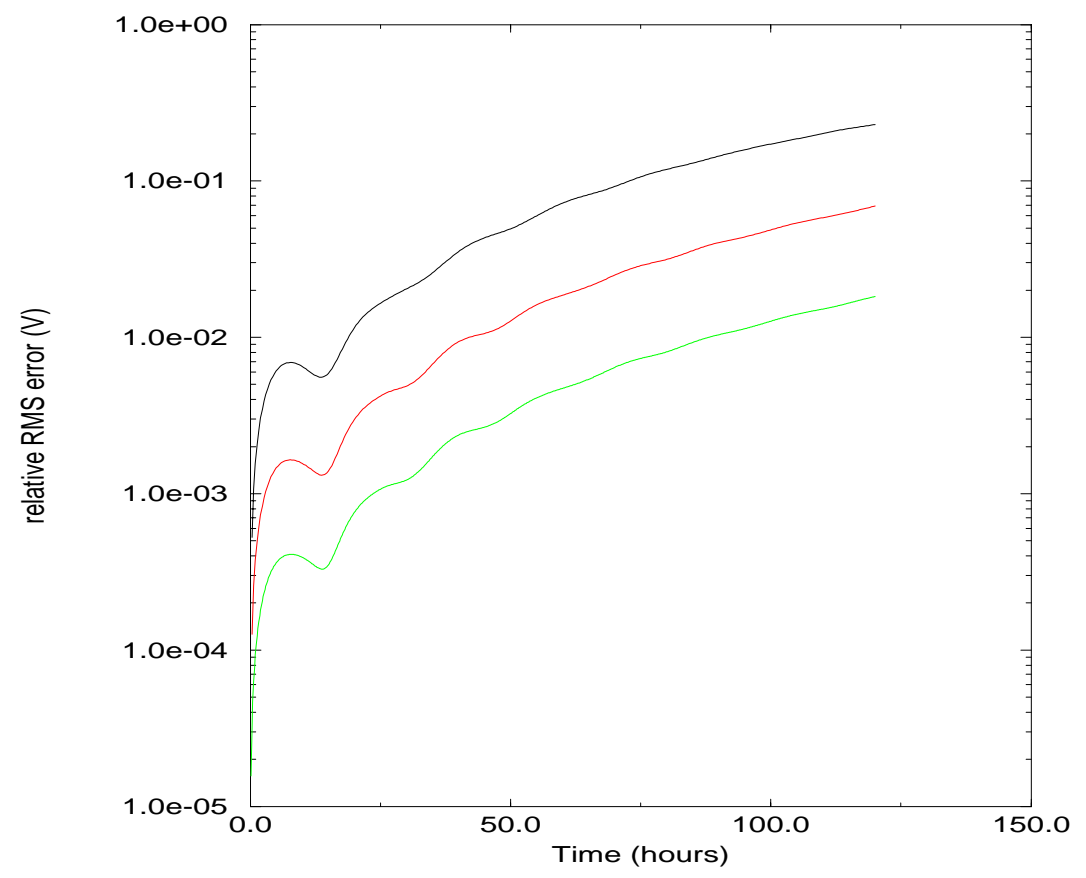

Fig. 4: Relative RMS Error in velocity; Test Case 2; $q=2,3,4$; Standard Form.

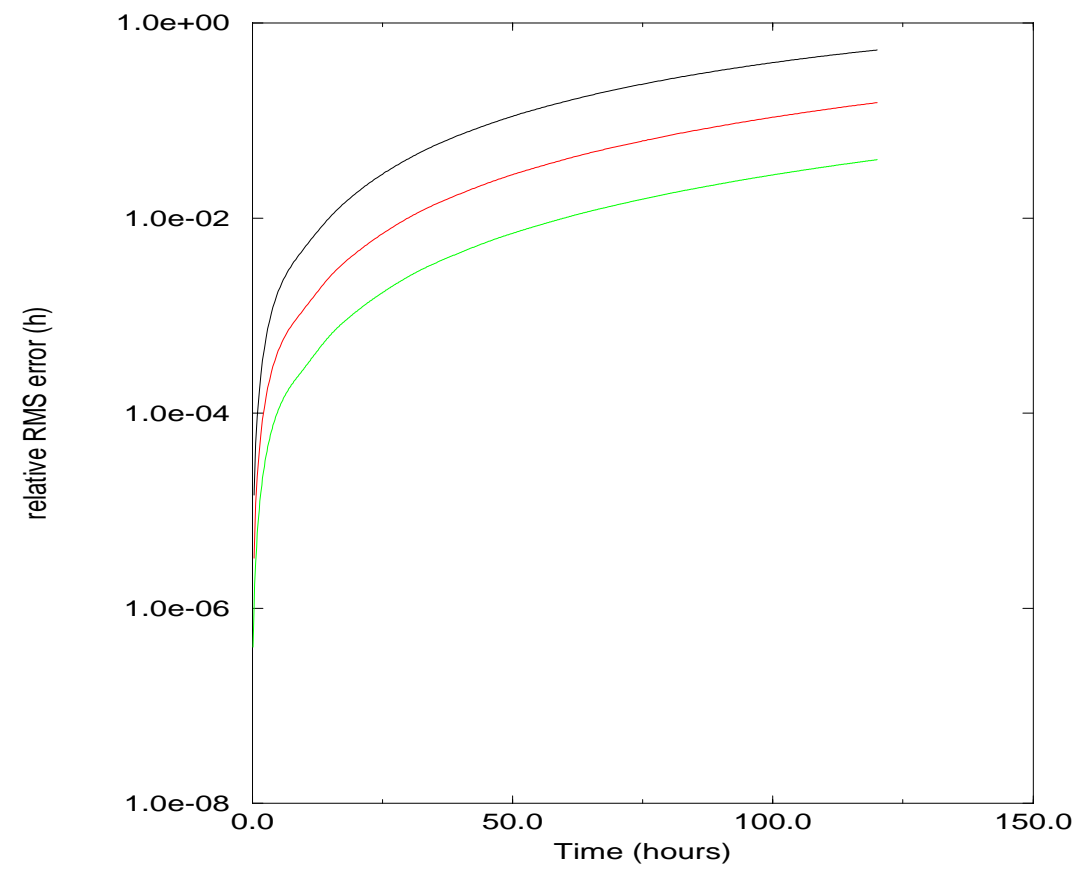

Fig. 5: Relative RMS Error in height; Test Case 2; $q=2,3,4$; Standard Form. 


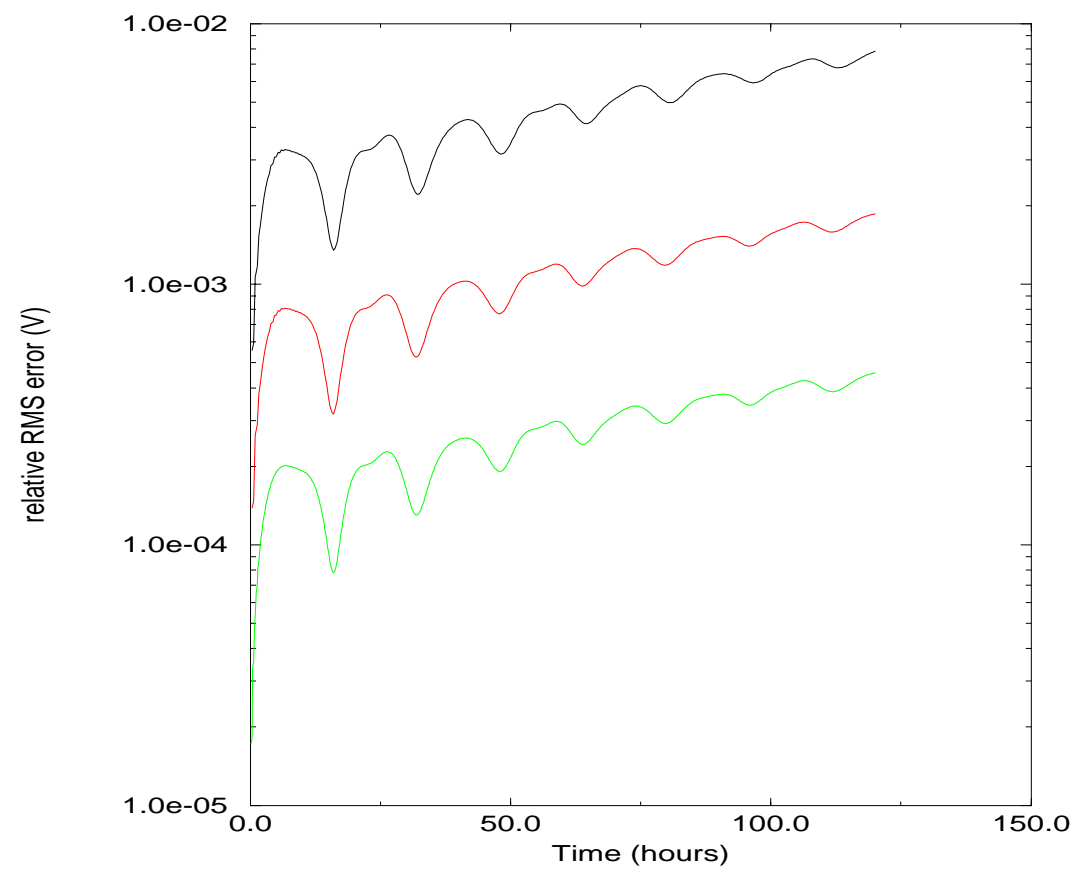

Fig. 6: Relative RMS Error in Velocity; Test Case 2; $q=2,3,4$; Rotational Form.

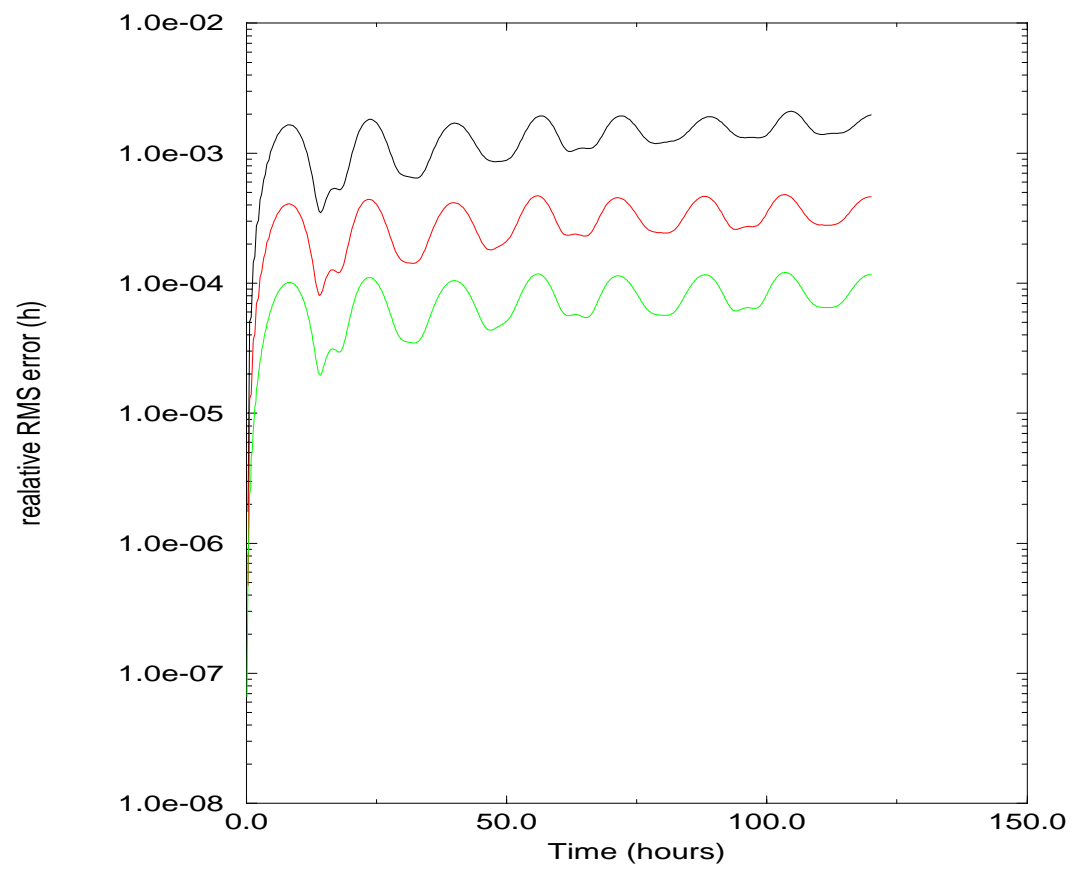

Fig. 7: Relative RMS Error in Height; Test Case 2; $q=2,3,4$; Rotational Form. 


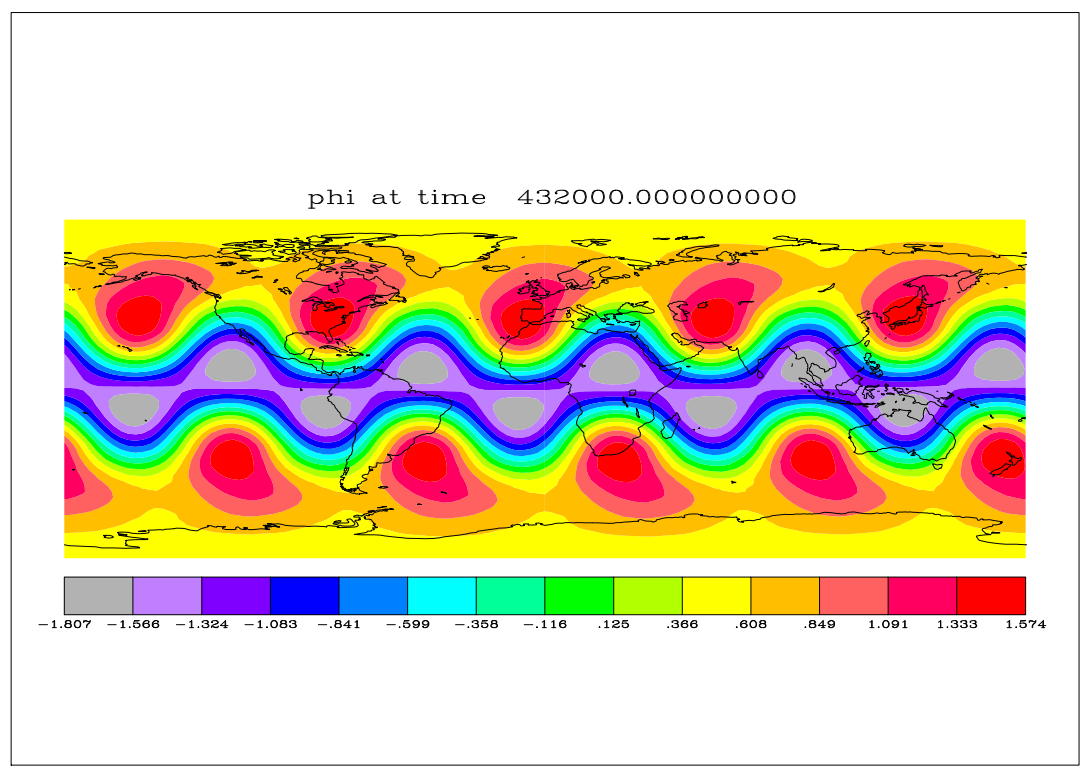

Fig. 8: Error in Geopotential at 5 days; Test Case 2; $q=4$; Rotational Form.

of this error in the nonlinear model due to a perturbation of the initial conditions? To explore this question the computed geopotential from the Cartesian model after one day was used as an initial condition in a spectral shallow water equation model, STSWM [4]. The spectral code was run at T42 resolution. After day five, the STSWM Fourier spectrum at a given latitude is compared to the spectrum of the Cartesian models solution for each day (Figure 9). The Fourier mode amplitude is the square of the modulus of the complex Fourier coefficient of the geopotential. The Fourier coefficients were sampled at the latitude of the T42 spectral model nearest 26.6 degrees, which is the location of one set of icosahedral points and the latitude of highest error in the geopotential.

The solution spectrums do not match but show the same features. The Cartesian solution has modes 5, 10, and 15 arising from the icosahedral points. These modes appear to grow over time in the Cartesian model, but no more so than the other modes. The perturbed spectral solution maintains the strong mode \#5 components at Day 5 with some redistribution of the other modes. The diffusion characteristics of the two methods seem evident. The continued growth of error in the Cartesian model might be attributed to the truncation errors injected in the solution at each timestep. The growth is not a nonlinear mode interaction since the perturbed spectral model shows a preservation of the mode 5 amplitude.

When the Cartesian solution is used as an initial condition in the spectral model, the errors are 


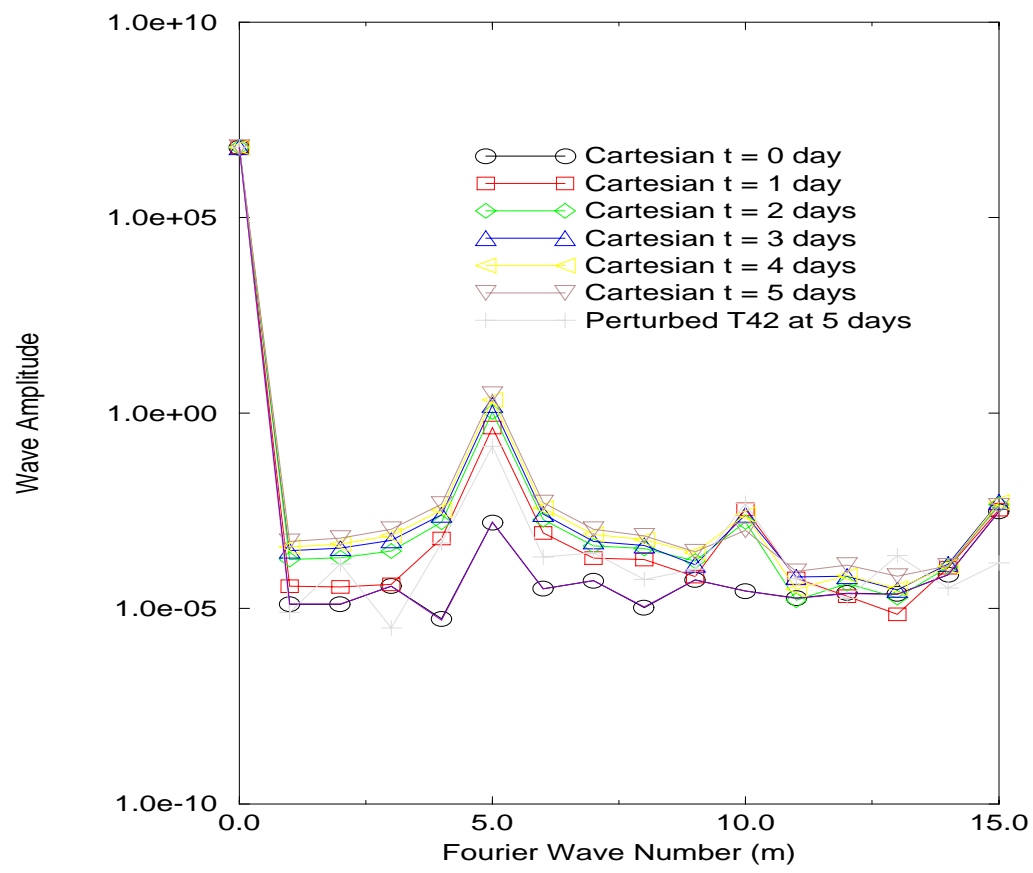

Fig. 9: Comparison of geopotential Fourier spectrum for Case 2; $q=3$; Rotational Form.

advected. As these errors (associated with different wave numbers) move around the globe, they sometimes cancel to produce smaller errors and other times superimpose to create larger errors. Figure 10 shows the error in the perturbed spectral solution for two different days in the northern hemisphere. The error on day 4 is considerably more dispersed and smaller than the error on Day 3 or Day 5, indicating a harmonic of the motion. The Cartesian model and the Heikes-Randall [5, 6] model both show eight peaks of error over the five day period. Once an error is present, it tends to remain over time, but each model exhibits different numerical diffusion. The Cartesian model shows a much smoother error than the spectral, indicating that it is more diffusive than the spectral model. The error profile of the Hiekes-Randall model ([6], Figure 9) also shows less diffusion than the Cartesian model. It should be reiterated that no diffusion has been explicitly added to any of these simulations for Case 2.

\subsection{NON-ANALYTIC, STEADY, ZONAL FLOW TEST}

Test Case 3 is a steady, non-linear zonal flow rotated through some angle $\alpha=\frac{\pi}{3}$. It is similar to Test Case 2, however, it contains compactly supported profiles not exactly representable by the spherical harmonics. The local spherical harmonic approximations for the derivative operators are able to capture the steady state solution but not as well as in Test Case 2. Figures 11 and 12 

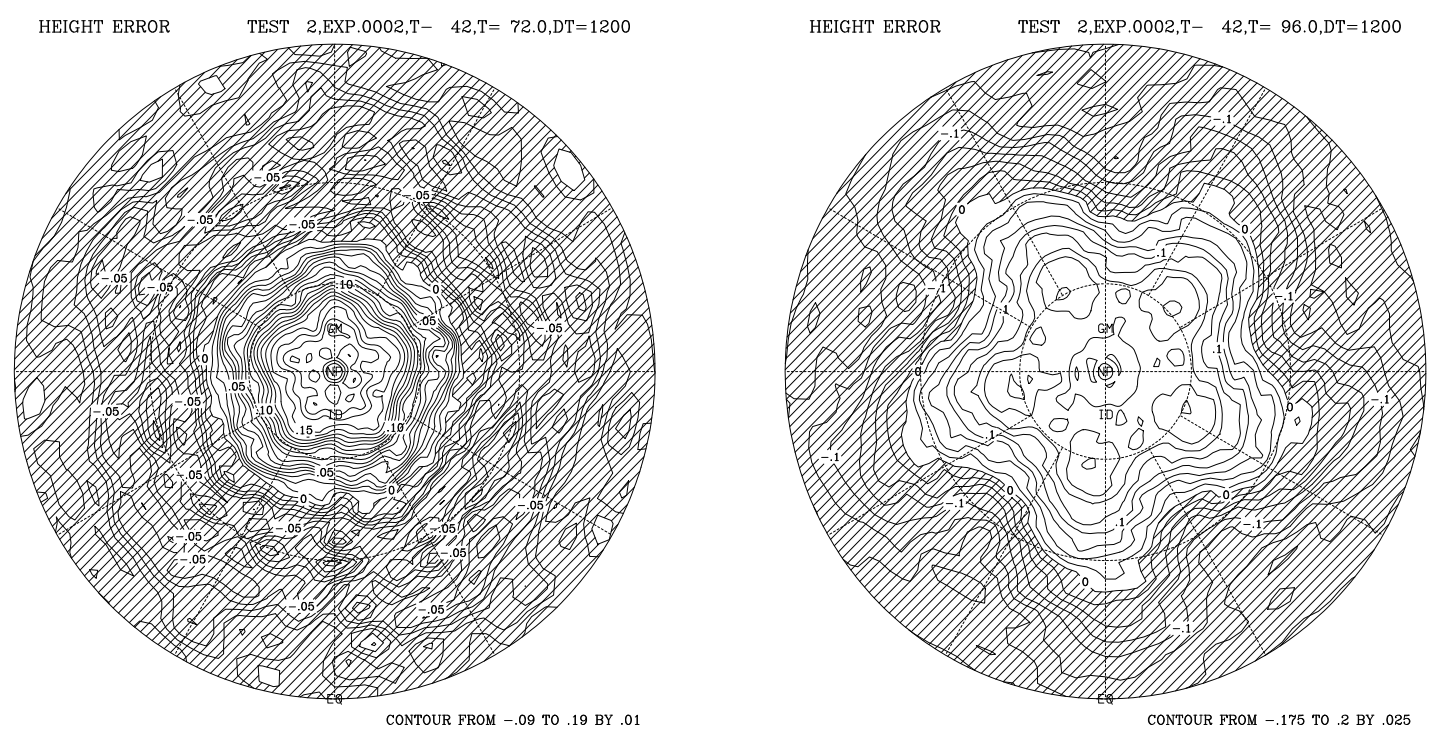

Fig. 10: Test Case 2, Perturbed Spectral Geopotential Error (a) at Day 4, (b) at Day 5.

show the error, as a function of time, in the velocity (using the relative RMS error with the exact steady solution) and the RMS error in the height field, respectively. Since the model includes no damping the error builds over time and then grows exponentially. The $q=2,3$ integrations used a time step of 1200 seconds for the 5-day (120 hour) simulations while the $q=4$ mesh used a 600 second timestep. For this case, no explicit diffusion was added to either the momentum or height equations.

The rotational form error results for Case 3 are given in Figures 13 and 14. A contour plot of the absolute geopotential error is given in Figure 15. The error is measured at Day 5.

\subsection{FORCED NONLINEAR SYSTEM WITH A TRANSLATING LOW}

Test Case 4 is a time-dependent, non-linear forced flow with an exact solution. It tests the performance of the scheme in an unsteady, dynamic simulation. The flow is a translating low pressure center superimposed on a jet stream symmetrical about the equator. The field is similar to a midlevel tropospheric flow with a short-wave trough embedded in a westerly jet. As the simulation progresses, the low translates eastward maintaining, its original shape.

Figures 16 and 17 show the error, as a function of time, in the velocity (using the relative RMS error with the exact solution) and the RMS error in the height field, respectively. The model in rotational form includes no damping. The $q=2,3$ integrations used a time step of 1200 seconds 


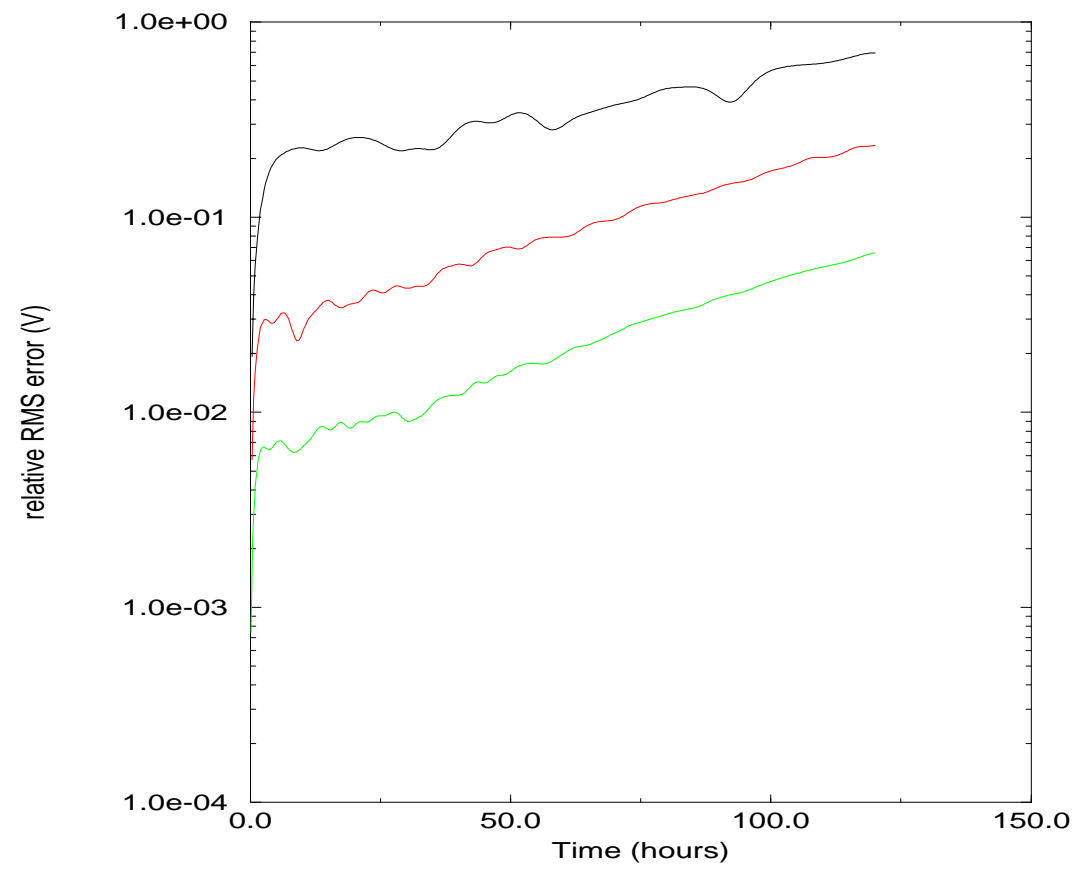

Fig. 11: Relative RMS Error in Velocity; Test Case 3; $q=2,3,4$; Standard Form.

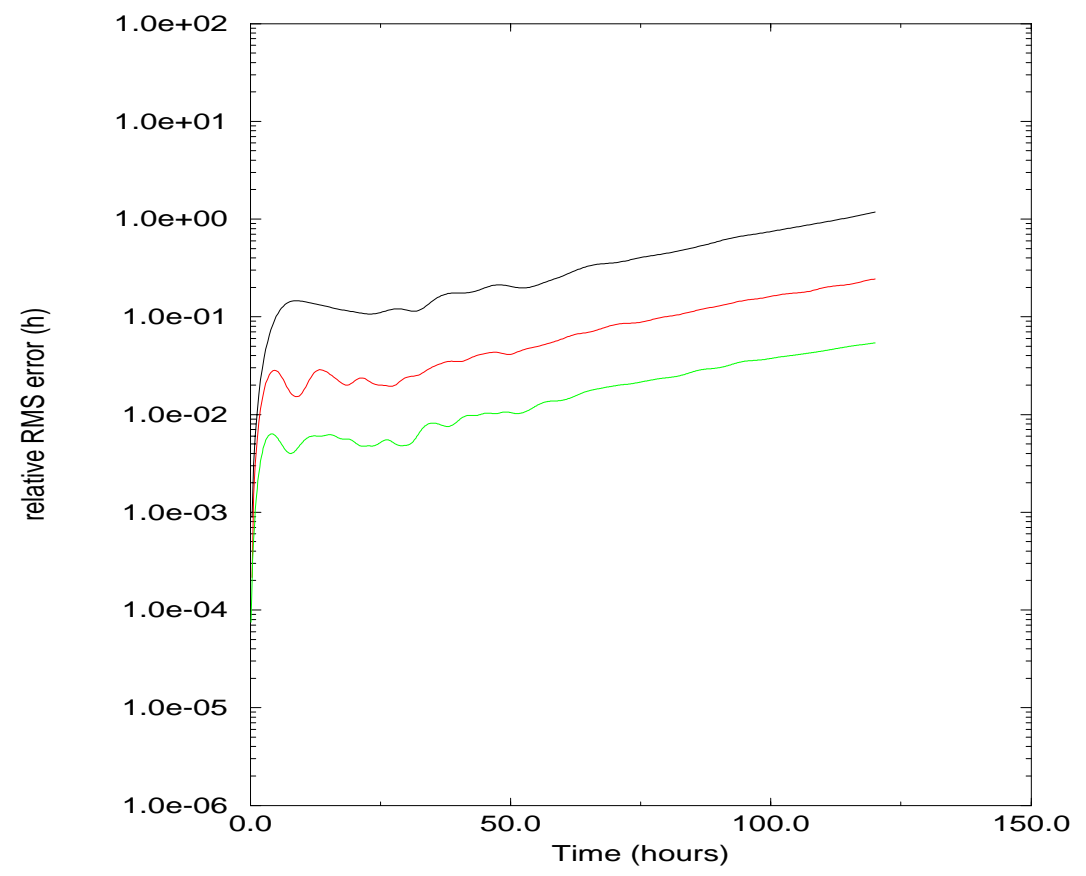

Fig. 12: Relative RMS Error in Height; Test Case 3; $q=2,3,4$; Standard Form. 


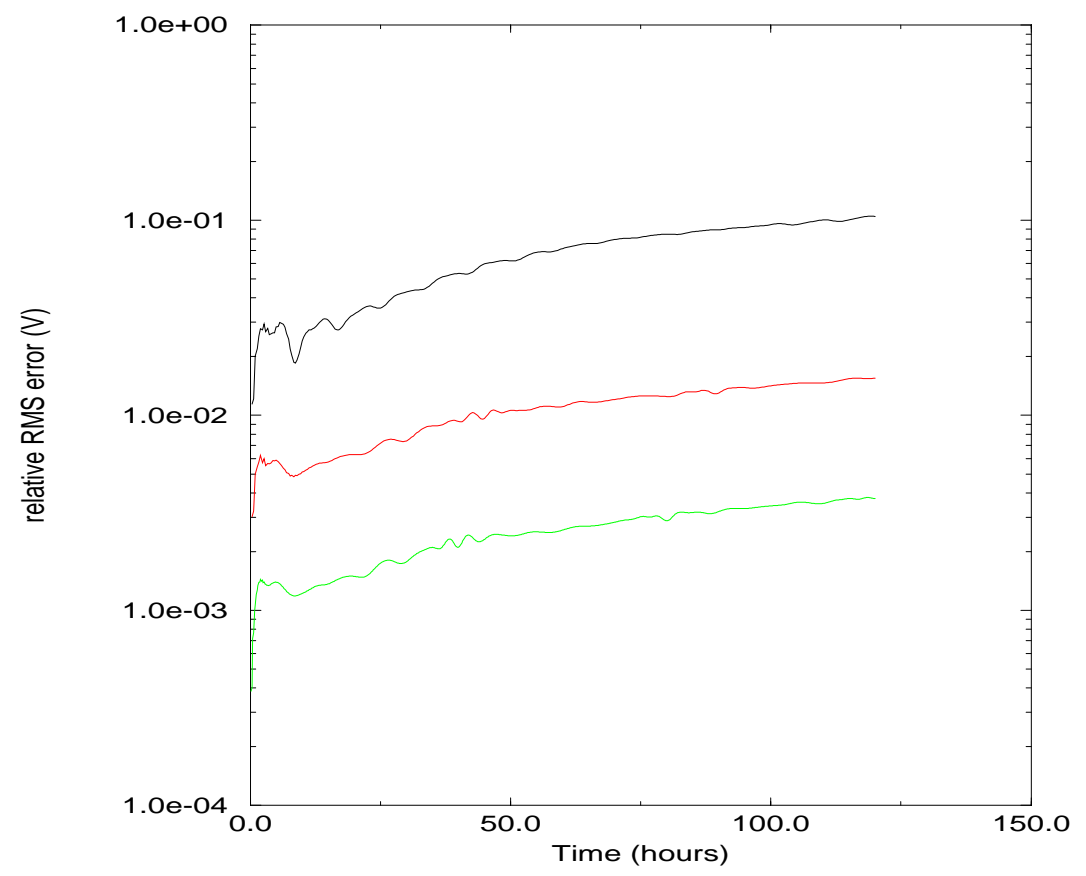

Fig. 13: Relative RMS Error in Velocity; Test Case 3; $q=2,3,4$; Rotational Form.

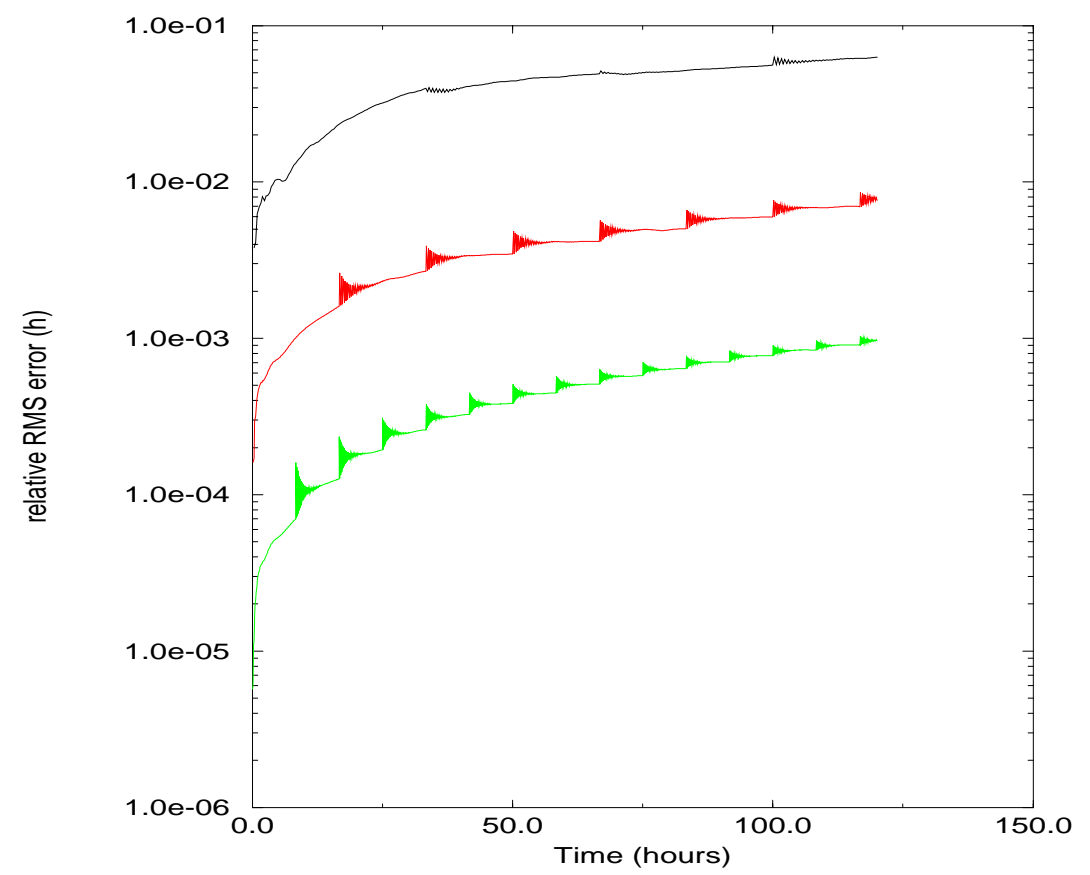

Fig. 14: Relative RMS Error in Height; Test Case 3; $q=2,3,4$; Rotational Form. 


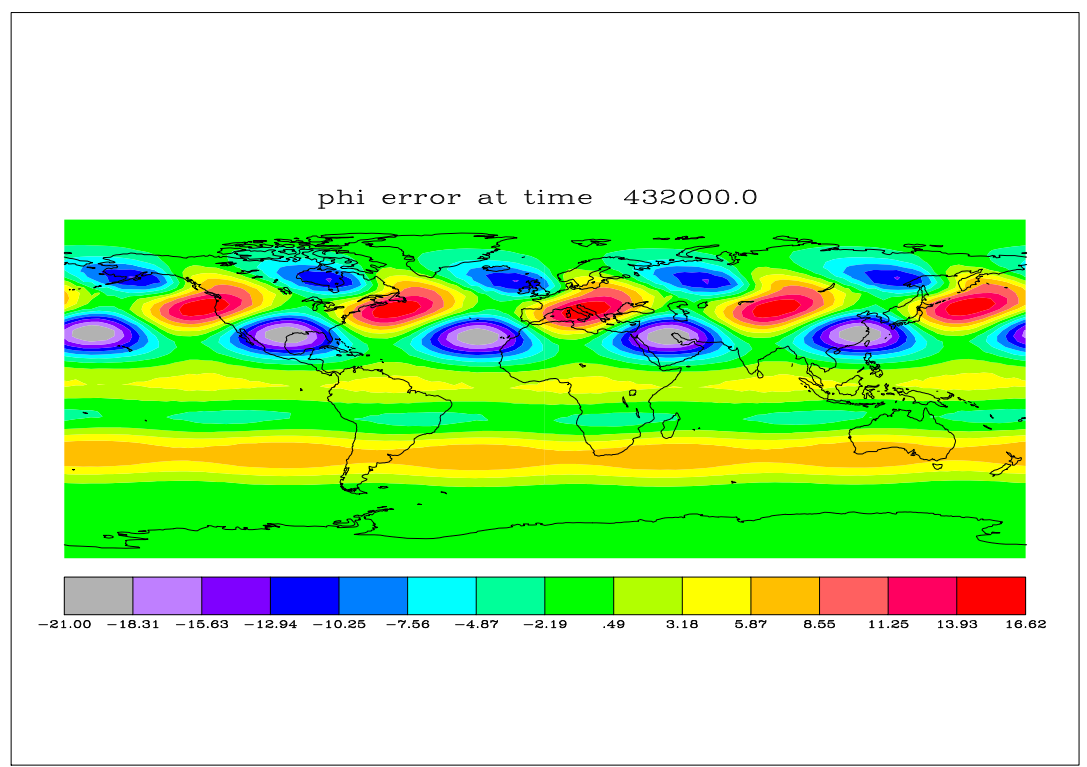

Fig. 15: Error in Geopotential at 5 days; Test Case 3; $q=4$; Rotational Form.

for the 5 day (120 hour) simulations while the $q=4$ mesh used a 600 second timestep. The convergence of the Cartesian method is again exhibited as the mesh is refined.

\subsection{ZONAL FLOW OVER AN ISOLATED MOUNTAIN}

This Test Case is the only one with orography. A 5400 meter high mountain is given through the surface height function, $h_{s}$. No analytical solution is known for this case so the usefulness of the case is in diagnosing the conservation properties of the numerical scheme. The simulation used a diffusion coefficient of $\varepsilon_{V}=5.0 \times 10^{5}$ with a timestep of 600 seconds for the $q=4$ mesh.

The following conserved quantities are presented as functions of time: mass, total energy, and potential enstrophy. The relative error of the conserved quantity is computed as a normalized integral of the quantity; the normalization is with respect to the integral of the initial value (see [24]). The vorticity is presented as an integral without normalization in Figure 18. The conservation properties of the Cartesian method are much better than expected, considering that the difference formula used to approximate the conservation of mass is not in flux form and are not guaranteed to preserve the global mass. The excellent conservation of enstrophy and vorticity are also a surprise. As normalized integrals it is not evident from Figure 18 that the integral of enstrophy maintains a value near machine zero $\left(\approx 10^{-13}\right)$ throughout the simulation. A contour plot of the geopotential 


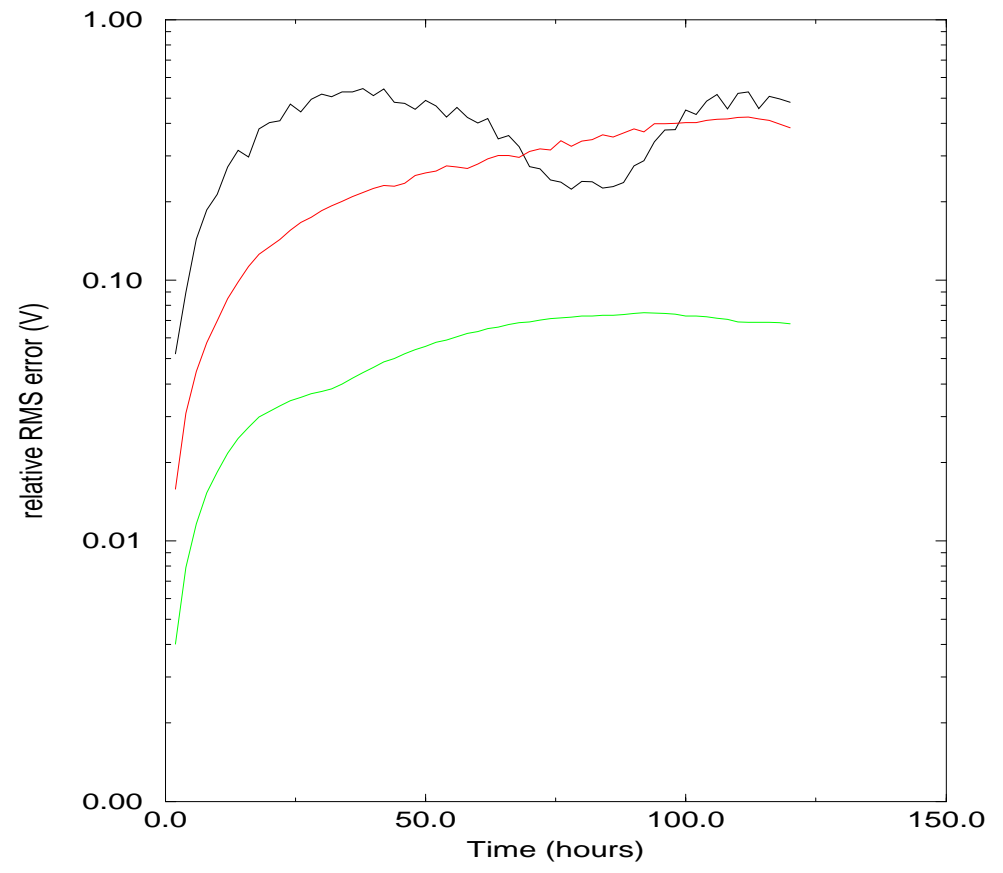

Fig. 16: Relative RMS Error in Velocity; Test Case 4; $q=2,3,4$; Rotational Form.

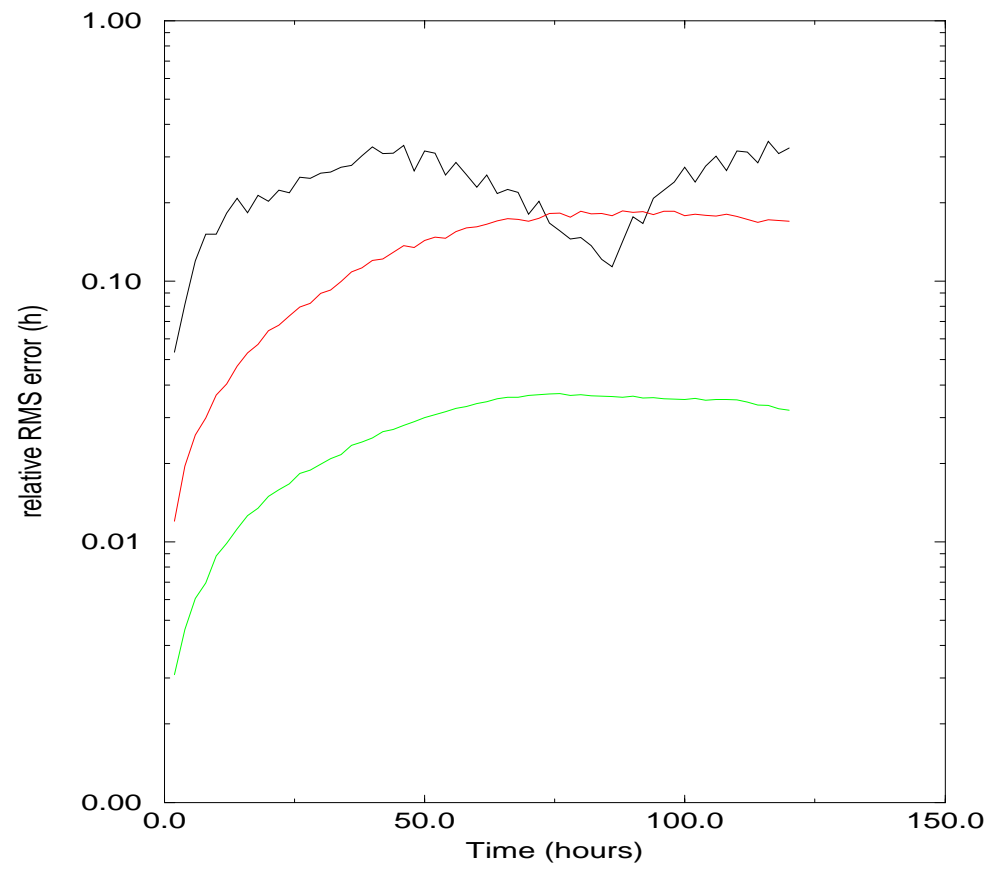

Fig. 17: Relative RMS Error in Height; Test Case 4; $q=2,3,4$; Rotational Form. 
$h^{*}$ at Day 15 is given in Figure 19.

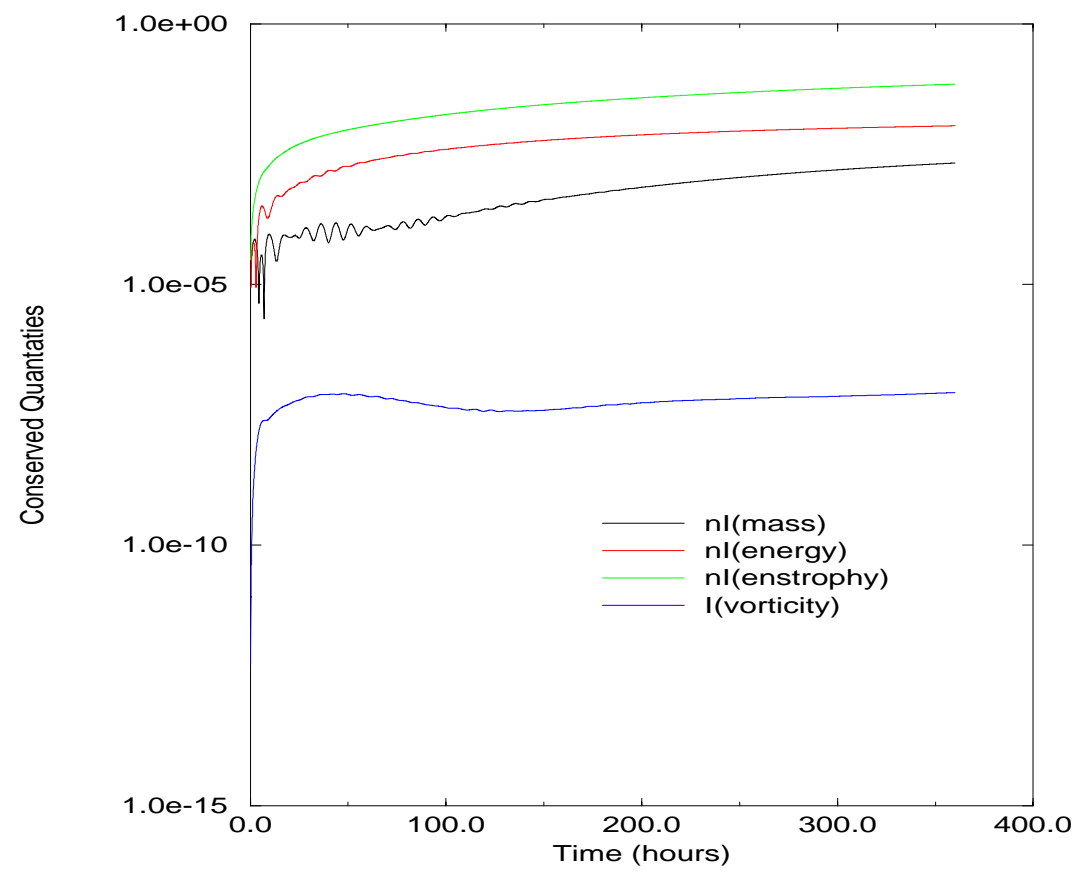

Fig. 18: Conserved integral quantities; Test Case 5; $q=4$; Rotational Form.

\subsection{ROSSBY-HAURWITZ WAVE}

This classic standard test case [10] does not have an analytic solution for the nonlinear shallow water equations. The initial conditions are zonal wave \#4. The solution at 0,7 , and 14 days continues to show the strong influence of the initial conditions. A diffusion coefficient of $\varepsilon_{V}=$ $1 \times 10^{5}$ was used for these calculations. Interestingly, this is close to the value of the diffusion coefficient used by Richardson [11] and also by Williamson [22].

The rotational form is semi-conservative of energy. Figure 20 shows the conserved quantities of mass, energy, and enstrophy as normalized integrals and the conserved integral of vorticity through the simulation. A contour plot of the geopotential after 14 simulated days is given in Figure 21 .

Departure of the solution from the initial wave \#4 is of interest. To examine the structure of the solution and whether a mode 5 influence is unduly impacting the Cartesian computation, the experiment using the spectral shallow water code (described in Test Case 2) was repeated. The Cartesian model geopotential output at Day 1 was used as an initial condition in a spectral shallow water equation model, STSWM [4]. For reference, the Cartesian solution used for input to the spectral model is plotted in Figure 22. After 5 days, the STSWM Fourier spectrum was compared 


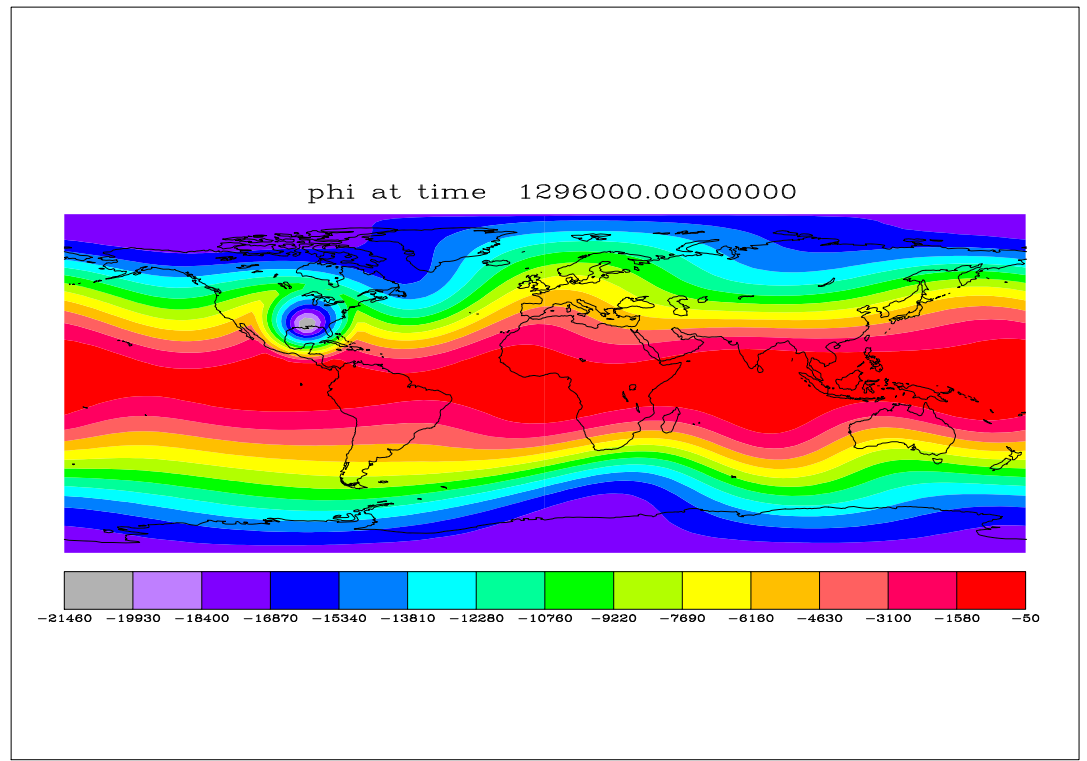

Fig. 19: Geopotential at 15 days; Test Case 5; $q=4$; Rotational Form.

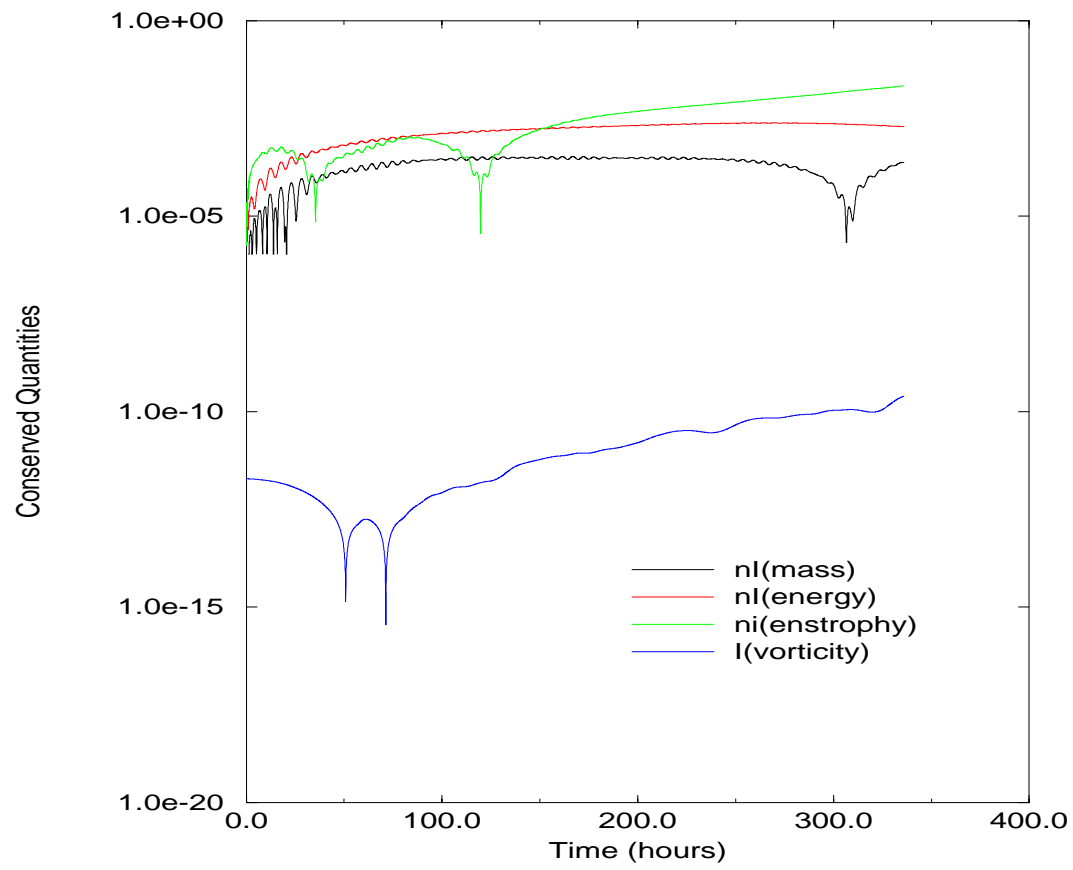

Fig. 20: Conserved integral quantities; Test Case 6; $q=4$; Rotational Form. 


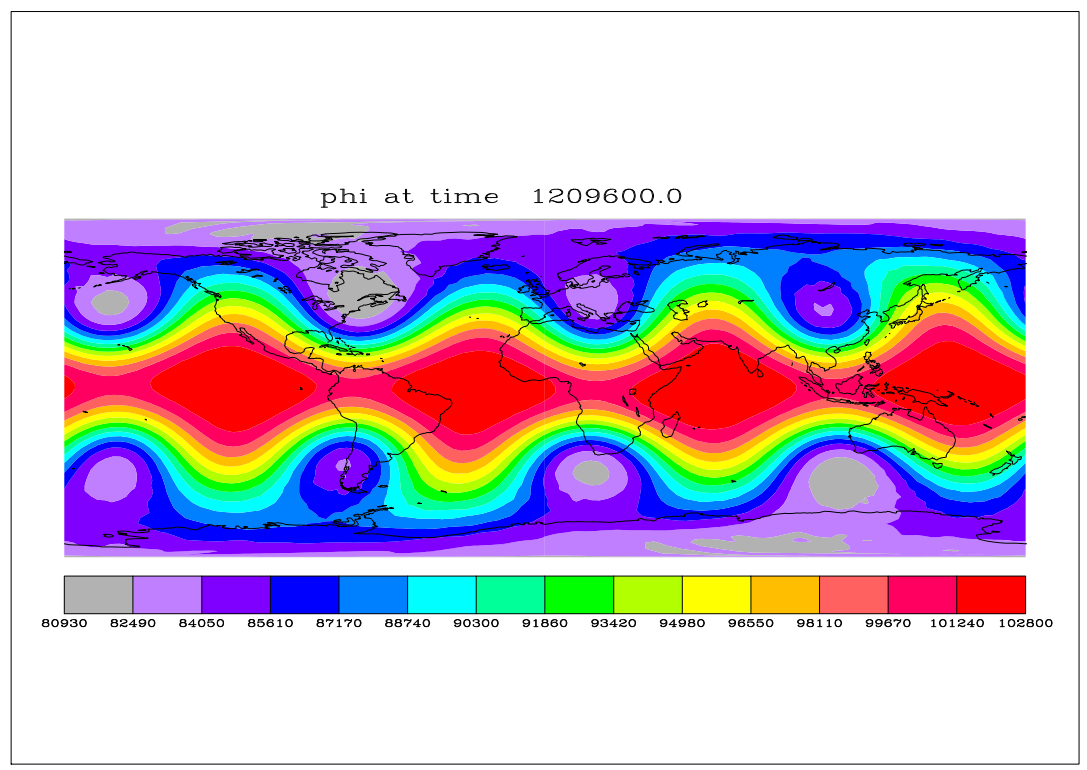

Fig. 21: Geopotential at 14 days; Test Case 6; $q=4$; Rotational Form.

to the spectrum of the Cartesian model solution at the Gaussian latitude nearest 50 degrees north in Figure 23.

The spectral analysis shows that the growth of low modes in the Cartesian model is similar to the growth in the spectral model. The low modes grow somewhat but are still three orders of magnitude less than wave \#4. Wave \#1-\#3 appear stable. Wave \#5-\#7 grow somewhat over the five days. In particular, the wave \#5 growth is only somewhat larger in the Cartesian model than in the spectral model. This indicates that the solution is exhibiting an interaction between modes in response to the perturbed initial conditions. The Cartesian method also shows a small compounding of truncation errors due to the icosahedral grid points in wave \#5. The distribution of spectral coefficients has more to do with the way energy cascades in the two models. This, in turn, is related to the diffusion of energy inherent in the models.

The solutions of the perturbed spectral model and the Cartesian model differ only slightly by the fifth day of the simulation. As seen in Figure 24, the Cartesian method exhibits a smoother solution due to the added diffusion. The asymmetry that develops in the perturbed spectral model by Day 14 is not evident, but the high latitude departure from wave \#4 is evident. This can be associated with the growth and interaction of the lower wave numbers. The wave 4 interacts with the wave 5 in the perturbation to produce a response in wave \#1 and wave \#9. These modes interact 


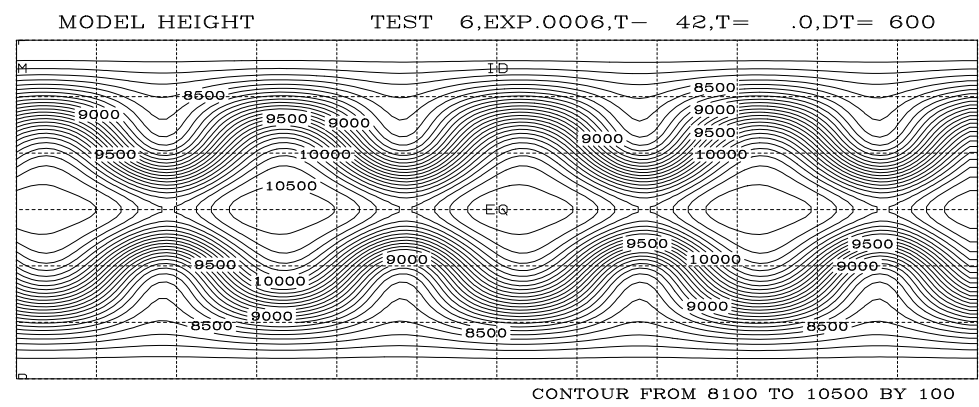

Fig. 22: Cartesian Geopotential at Day 1.

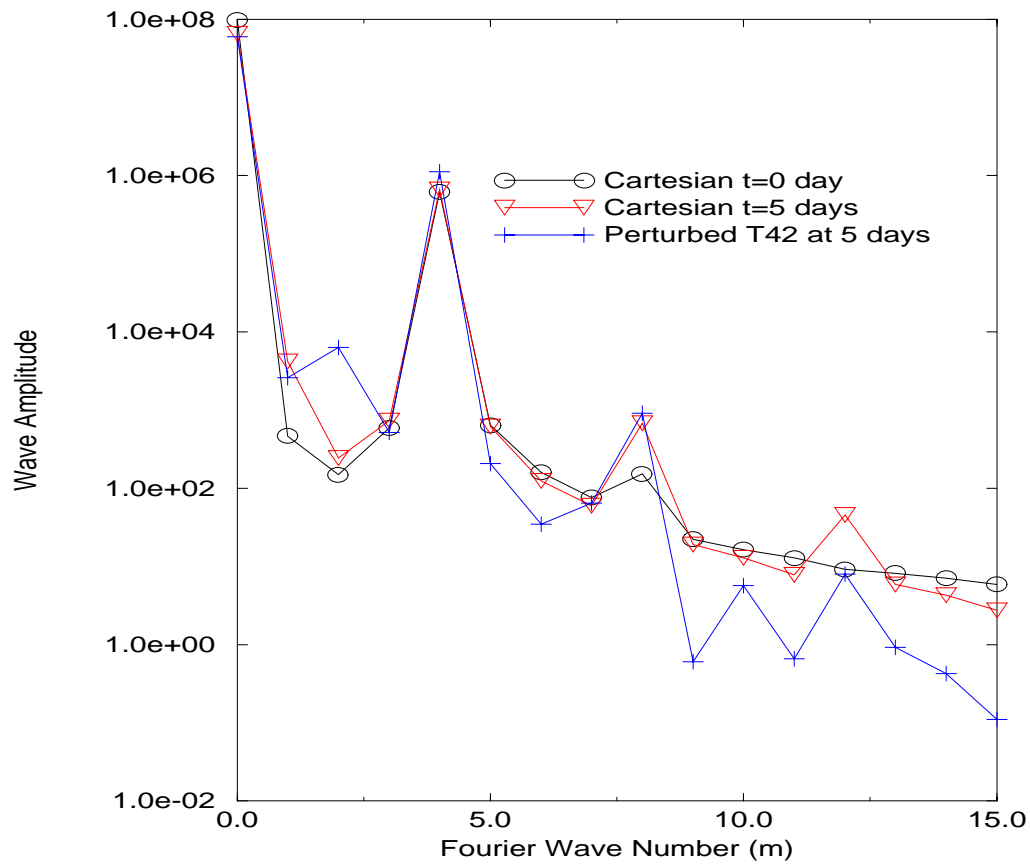

Fig. 23: Comparison of geopotential Fourier spectrum for Case 6; $q=3$; Rotational Form. 
with the entire spectrum.


Fig. 24: Test Case 6, (a) Cartesian Geopotential at Day 5, (b) Perturbed Spectral Geopotential at Day 5.

\subsection{ANALYZED 500mb INITIAL CONDITIONS}

The real data initial conditions differ in smoothness from the previous cases, exhibiting much finer scale structure and sharper gradients. For non-linear calculations, there will be a much stronger interaction between modes and more active dispersive phenomena. A diffusion coefficient (the same as for Test Case 6) controls the build up of energy in the fine scales. A timestep of 300 seconds is used on the $q=4$ mesh with 10,242 points.

The first test case using analyzed atmospheric conditions is for 000GMT 21 December 1978. The spectral non-linear normal mode analysis has been used to filter gravity waves from this data. The NCAR netCDF file "REF0077.cdf" was used for initial conditions of both geopotential and velocity at the icosahedral grid points. The strong flow over the north pole has been useful in diagnosing pole problems for several numerical schemes.

Figures 25 shows the conserved quantities of mass, energy, and enstrophy as normalized integrals and the conserved integral of vorticity through the simulation. Figures 26 and 27 show a comparison of the reference solution with the Cartesian solution at one and five days, respectively. 


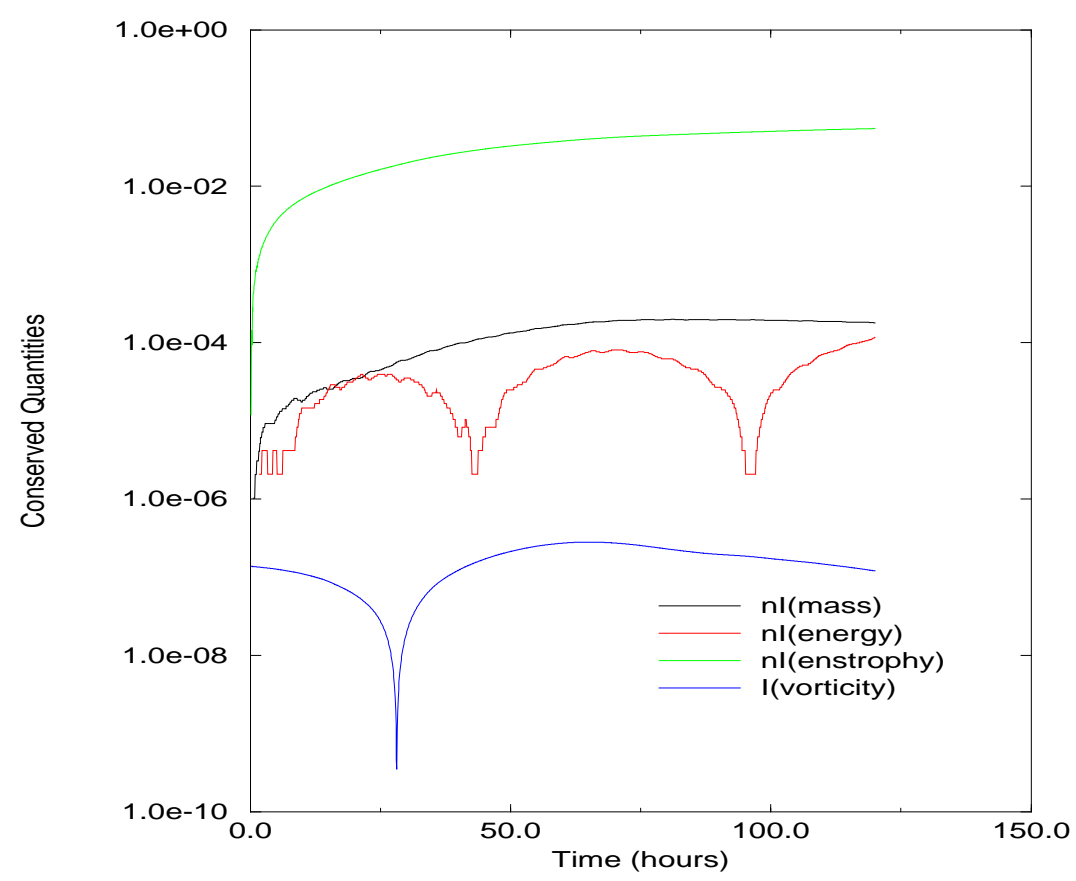

Fig. 25: Conserved integral quantities; Test Case 7a; $q=4$; Rotational Form.
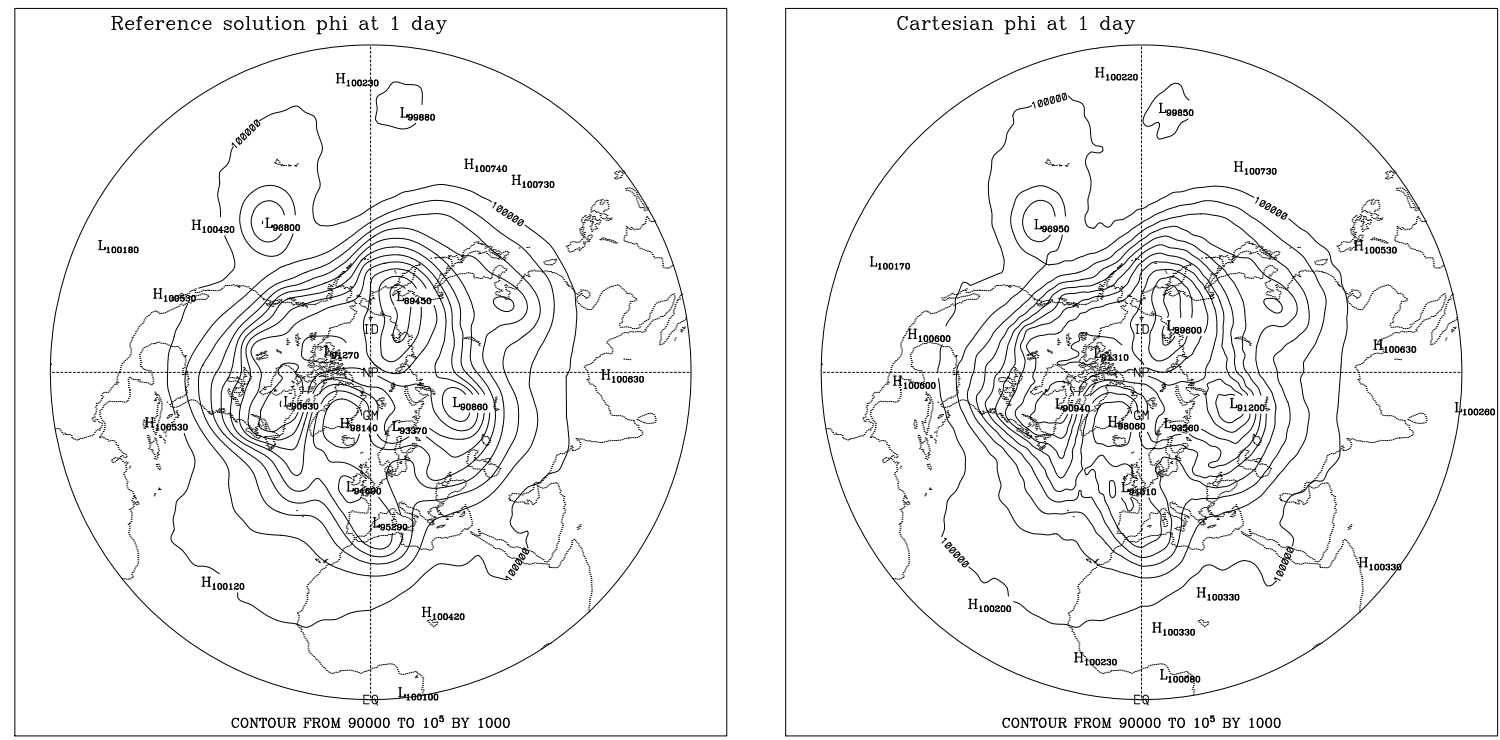

Fig. 26: Test Case 7a, (a) Reference Solution at Day 1, (b) Cartesian Geopotential at Day 1. 

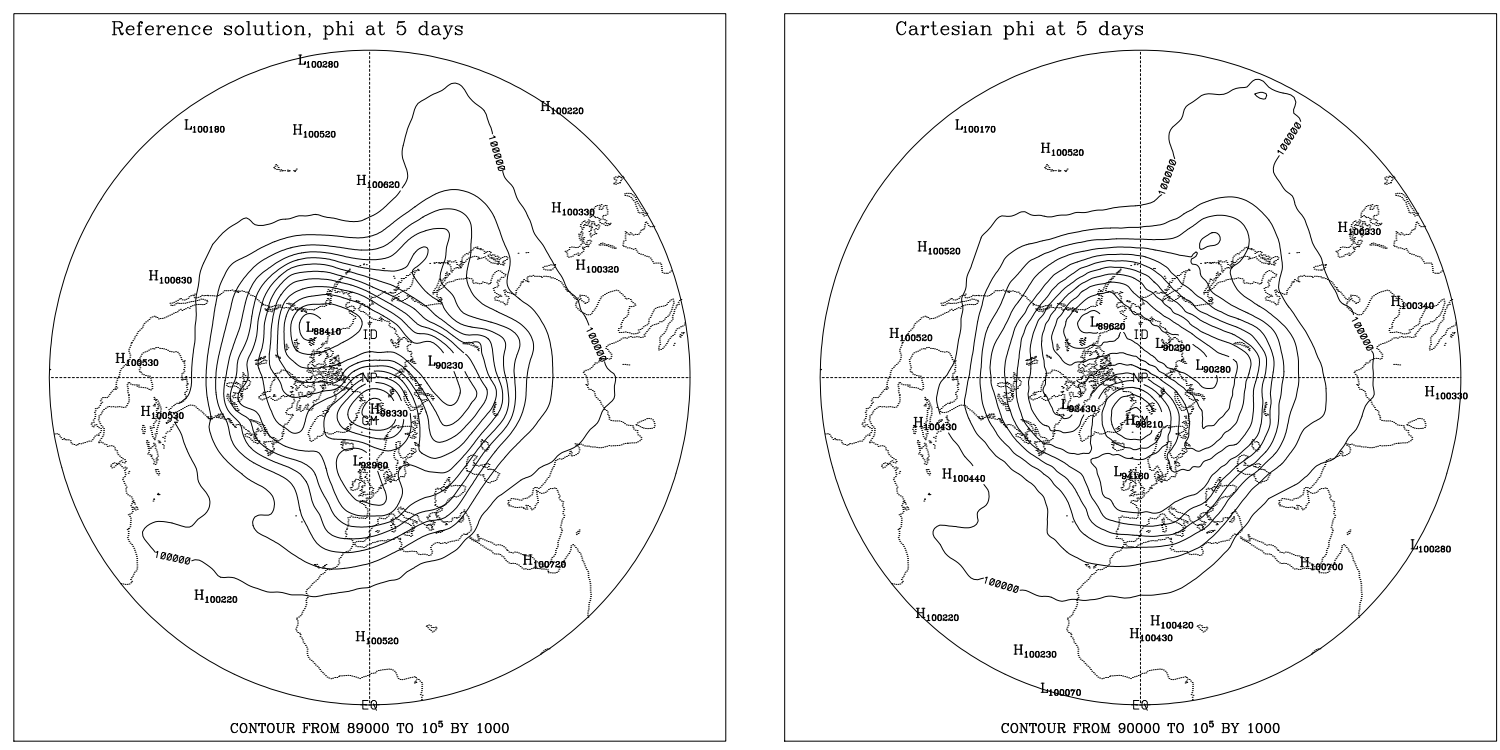

Fig. 27: Test Case 7, (a) Reference Solution at 5 Day, (b) Cartesian Geopotential at 5 Day.

The second real data case uses the NCAR file "REF0087.cdf". The flow develops into a typical blocking situation from an initial condition of two cut-off lows. Initial conditions are for 0000 GMT 16 January 1979. Figures 28 and 29 show a comparison of the reference solution with the Cartesian solution at Day 1 and Day 5, respectively.

Figures 30 shows the conserved quantities of mass, energy, and enstrophy as normalized integrals and the conserved integral of vorticity through the simulation.

The third real data case has initial conditions from 0000 GMT 9 January 1979 and uses the NCAR file "REF0088.cdf". Initially it has a strong zonal flow. Figures 31 and 32 show a comparison of the reference solution with the Cartesian solution at Day 1 and Day 5, respectively.

Figures 33 shows the conserved quantities of mass, energy, enstrophy as normalized integrals and the conserved integral of vorticity through the simulation.

\section{CONCLUSIONS}

Numerical methods for shallow water equations on the sphere are faced with three hurdles. First, the geostrophic wind balance must be maintained accurately. The numerical approximation for the gradient of the geopotential must balance the Coriolis term well. Since the Coriolis term does not involve derivatives, the gradient approximation is crucial to achieve a reasonable balance. Second, 

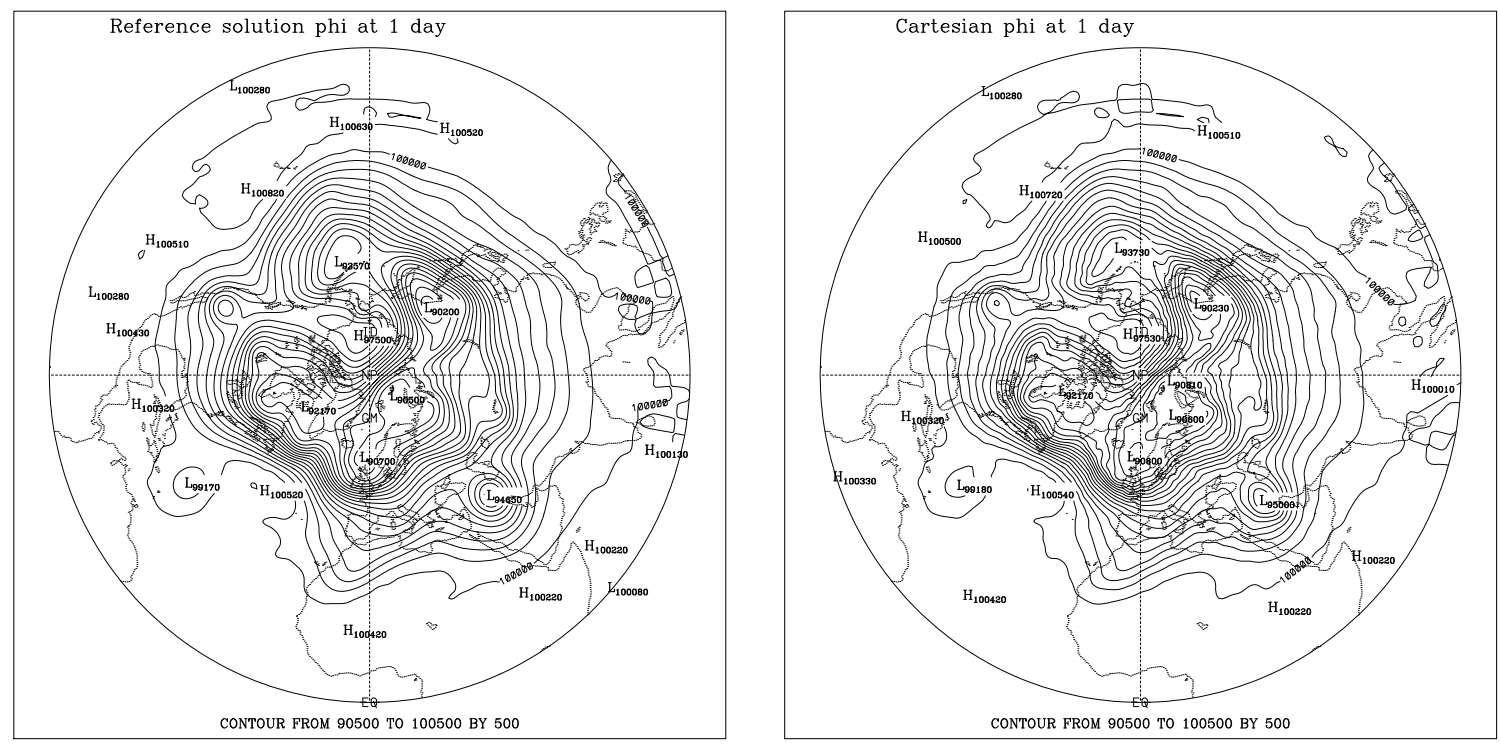

Fig. 28: Test Case 7b, (a) Reference Solution at Day 1, (b) Cartesian Geopotential at Day 1.
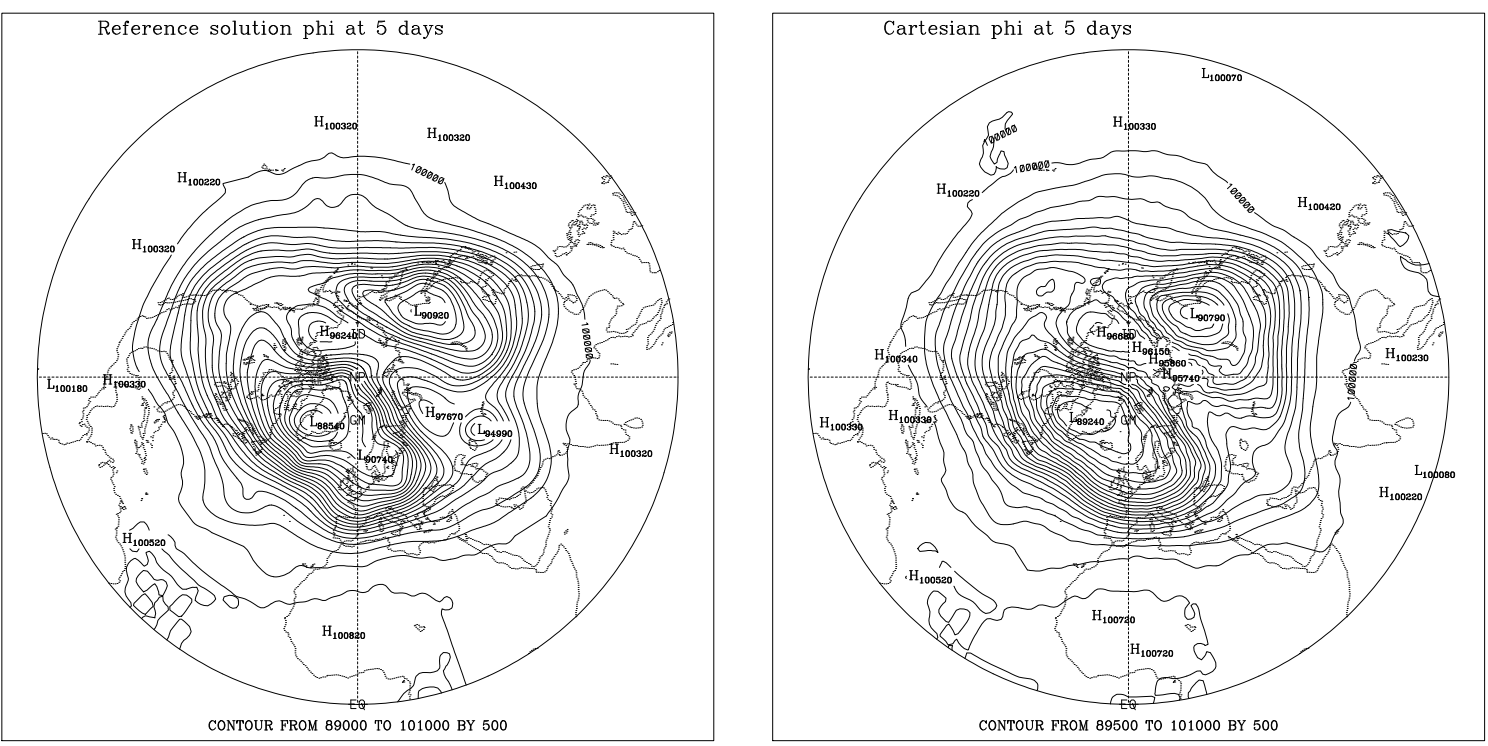

Fig. 29: Test Case 7, (a) Reference Solution at 5 Day, (b) Cartesian Geopotential at 5 Day. 


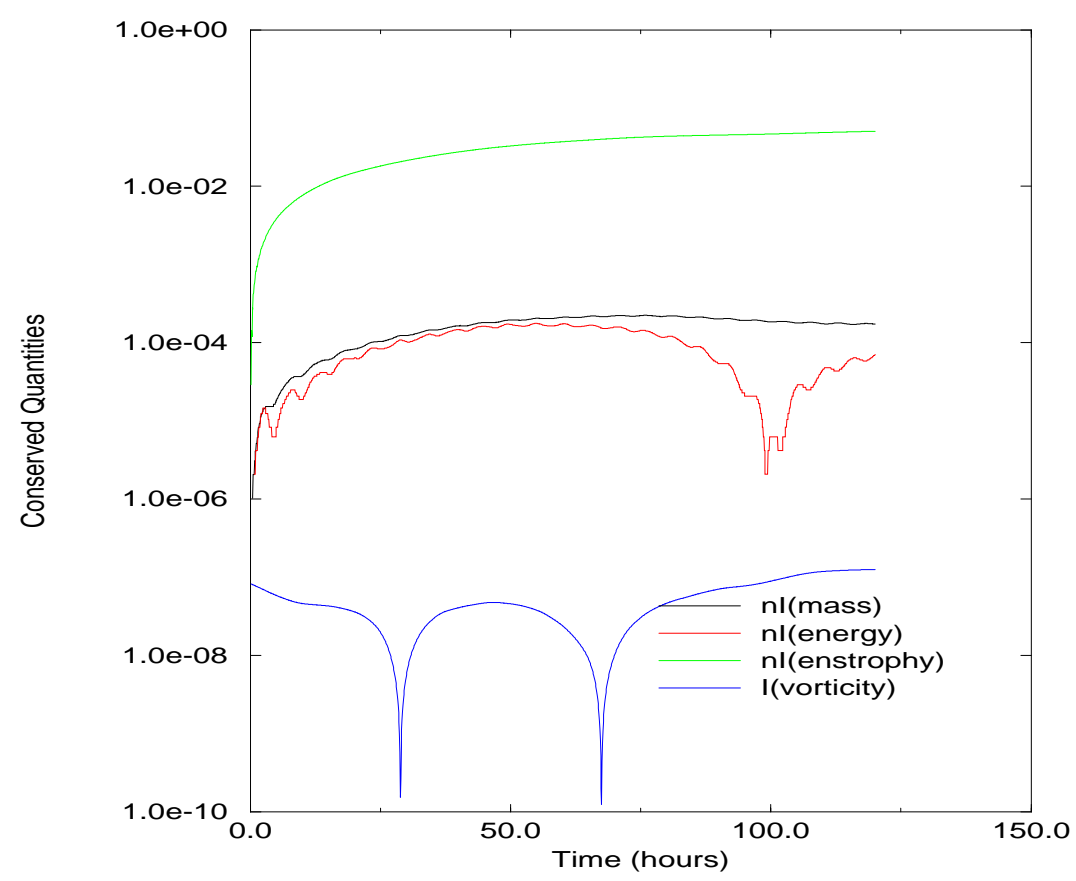

Fig. 30: Conserved integral quantities. Test Case 7b, $q=4$; Rotational Form.
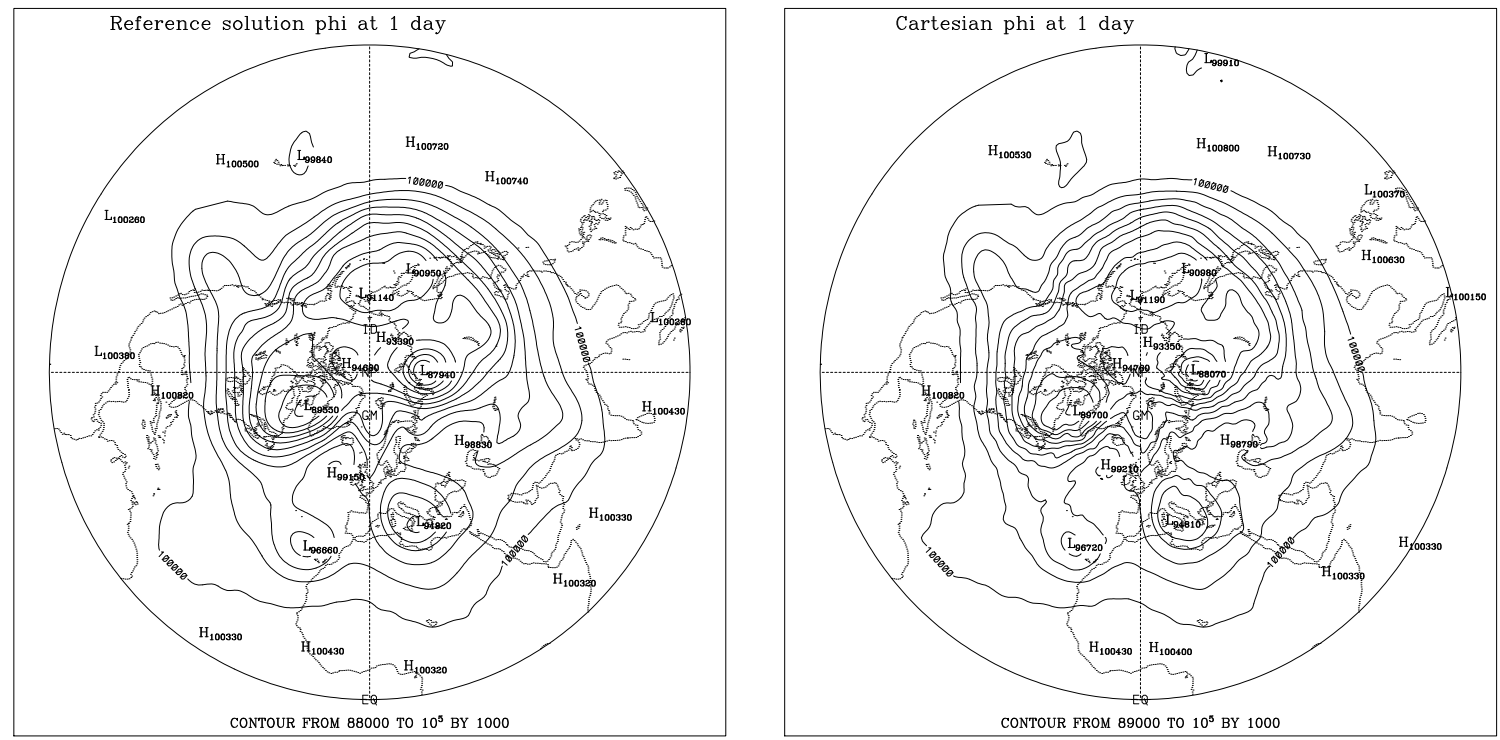

Fig. 31: Test Case 7c, (a) Reference Solution at Day 1, (b) Cartesian Geopotential at Day 1. 

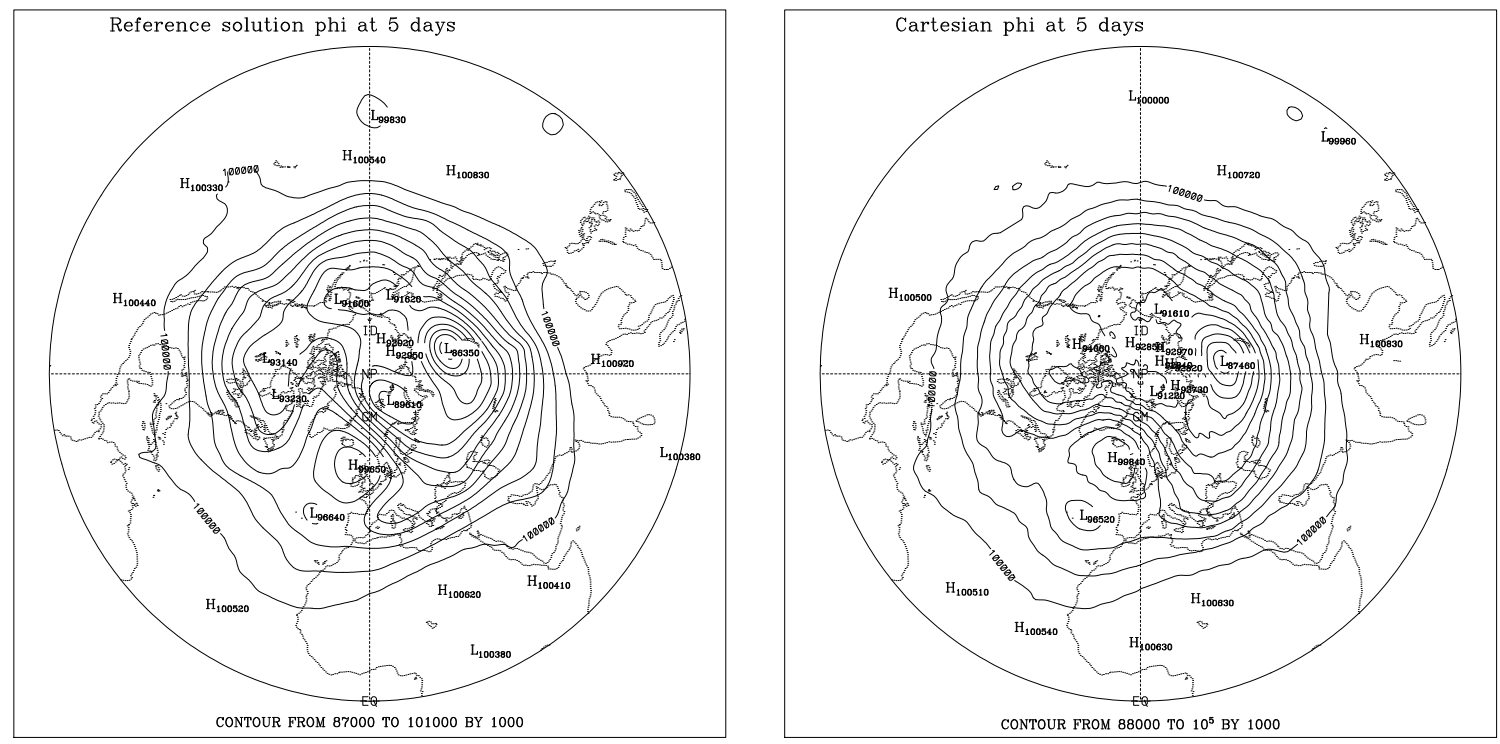

Fig. 32: Test Case 7c, (a) Reference Solution at 5 Day, (b) Cartesian Geopotential at 5 Day.

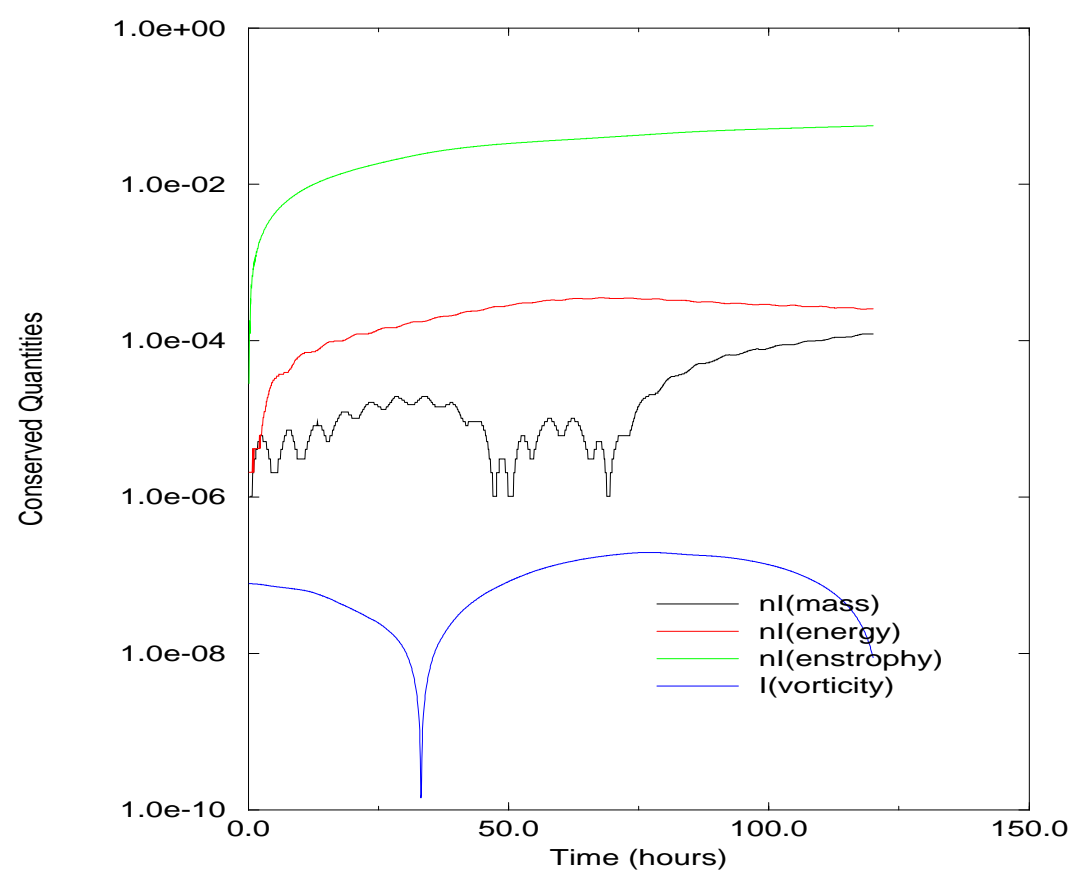

Fig. 33: Conserved integral quantities. Test Case 7c, $q=4$; Rotational Form. 
the pole problem must be addressed. Since the spherical coordinate representation of the velocity is singular at the poles, derivatives must be approximated with care. The third hurdle is to maintain stability. As nonlinear equations, the consistency of energy conversion terms from potential to kinetic energy plays a crucial role.

In our work, the first hurdle has been passed by adopting a co-located velocity and gradient approximation. The collocation method with accurate approximations for the derivatives gives an excellent balance of the geostrophic wind terms. The second hurdle is passed because the Cartesian method is free of the pole problem with continuous velocities at the poles. Finally, the third hurdle is addressed because the rotational form of the equations in Cartesian form gives reasonably good energy conservation to maintain stability.

The use of least squares fitting of the quadratic spherical harmonics to a seven point stencil has given second order convergence for the chosen cases. It has also been pointed out [3] that the Cartesian method does not require the solution of elliptic equations and has a low operation count with an easy parallel implementation. The deficiencies of the Cartesian method in comparison with other icosahedral mesh methods like Hiekes and Randall's [6], are the lack of exact conservation of enstrophy and a more diffusive scheme. As an advection scheme the Cartesian method suffers from the same lack of monotonicity as the spectral method.

Future work will involve the testing of higher order Cartesian discretizations and the extension to a 3-dimensional baroclinic model. 


\section{References}

[1] C. Canuto, M.Y. Hussaini, A. Quarteroni, and T.A. Zang. Spectral Methods in Fluid Dynamcis. Springer-Verlag, Berlin, 1988.

[2] I-Liang Chern. A Control Volume Method on a Icosahedral Grid for Numerical Integration of the Shallow-water Equations on the Sphere . MCS preprint, Argonne National Laboratory, Chicago, IL, 1991.

[3] J.B. Drake and M.P. Coddington. A Parallel Performance Study of the Cartesian Method for Partial Differentail Equations on a Sphere. ORNL Tech Report ORNL/TM-13304, Oak Ridge National Laboratory, Oak Ridge, TN, 1997.

[4] J.J. Hack and R. Jakob. Description of a Global Shallow Water Model Based on the Spectral Transform Method. NCAR Technical Note NCAR/TN-343+STR, National Center for Atmospheric Research, Boulder, CO, 1992.

[5] Ross Heikes and David A. Randall. Numerical Integration of the Shallow-Water Equations on a Twisted Icosahedral Grid. Part I. Mon. Wea. Rev., 123:1862-1880, 1995.

[6] Ross Heikes and David A. Randall. Numerical Integration of the Shallow-Water Equations on a Twisted Icosahedral Grid. Part II. Mon. Wea. Rev., 123:1881-1887, 1995.

[7] C.L. Lawson and R.J. Hanson. Solving Least Squares Problems. Prentice-Hall, Englewood Cliffs, NJ, 1974.

[8] B. Machenhauer. The spectral method. In Numerical Methods Used in Atmospheric Models, volume II of GARP Pub. Ser. No. 17. JOC, chapter 3, pages 121-275. World Meteorological Organization, Geneva, Switzerland, 1979.

[9] Y. Masuda and H. Ohnishi. An Integration Scheme of the Primitive Equation Model with an Icosahedral-Hexagonal Grid System and its Application to the Shallow Water Equations. In Short- and Medium-Range Numerical Weather Prediction, pages 317-326, 1986.

[10] N. A. Phillips. Numerical Integration of the Primitive Equations on the Hemisphere. Mon. Wea. Rev., 87:333-345, 1959. 
[11] L.F. Richardson. Atmospheric Diffusion Shown on a Distance Neighbour Graph. Proc. Roy. Soc. Ser. A, 110:709-737, 1928.

[12] R. Sadourny, A. Arakawa, and Y. Mintz. Integration of the Nondivergent Barotropic Vorticity Equation with an Icosahedral-Hexagonal Grid for the Sphere. Mon. Wea. Rev., 96:351-356, 1968.

[13] R. Sadourny and P. Morel. A Finite-Difference Approximation of the Primitive Equations for a Hexagonal Grid on a Plane. Mon. Wea. Rev., 97:439-445, 1969.

[14] I. A. Stegun. Legendre functions. In M. Abramowitz and I. A. Stegun, editors, Handbook of Mathematical Functions, chapter 8, pages 332-353. Dover Publications, New York, 1972.

[15] P. N. Swarztrauber. The Approximation of Vector Functions and Their Derivatives on the Sphere. SIAM J. Numer. Anal., 18:191-210, 1981.

[16] P.N. Swarztrauber. Spectral Transform Methods for Solving the Shallow-Water Equations on the Sphere. Mon. Wea. Rev., 124(4):730-744, 1996.

[17] P.N. Swarztrauber, D.L. Williamson, and J.B. Drake. The Cartesian Method for Solving of PDE's in Spherical Geometry. Dyn. Atm. Ocn., 27:679-706, 1997.

[18] A.N. Tikhonov and A.A. Samarskii. Equations of Mathematical Physics. Dover Publications, New York, 1963.

[19] D. L. Williamson. Integration of the Barotropic Vorticity Equation on a Spherical Geodesic Grid. Tellus, 20:642-653, 1968.

[20] D. L. Williamson. Integration of the Primitive Barotropic Model Over a Spherical Geodesic Grid. Mon. Wea. Rev., 98:512-520, 1969.

[21] D. L. Williamson. Numerical Integration of Fluid Flow Over Triangular Grids. Mon. Wea. Rev., 97:885-895, 1969.

[22] D. L. Williamson. A Comparison of First- and Second-Order Difference Approximations over a Spherical Geodesic Grid. J. Comp. Phys., 7:301-309, 1971. 
[23] D. L. Williamson. Numerical Methods Used in Atmospheric Models, chapter 2, pages 51120. GARP Pub. Ser. No. 17. JOC, WMO, Geneva, Switzerland, 1979.

[24] D. L. Williamson, J.B. Drake, J.J. Hack, Rudiger Jakob, and P.N. Swarztrauber. A Standard Test Set for Numerical Approximations to the Shallow Water Equations on the Sphere. J. Comp. Phys., pages 211-224, 1992. 


\section{A SPHERICAL HARMONIC POLYNOMIALS}

$n=0$ :

$$
Y_{0}^{0}=1
$$

$n=1:$

$$
\begin{aligned}
& r Y_{1}^{0}=z \\
& r Y_{1}^{1}=-x-\mathbf{i} y
\end{aligned}
$$

$n=2$ :

$$
\begin{aligned}
r^{2} Y_{2}^{0} & =\frac{3}{2} z^{2}-\frac{1}{2} \\
r^{2} Y_{2}^{1} & =-3(x+\mathbf{i} y) z \\
r^{2} Y_{2}^{2} & =3 x^{2}+6 \mathbf{i} x y-3 y^{2}
\end{aligned}
$$

$n=3$ :

$$
\begin{aligned}
r^{3} Y_{3}^{0} & =z^{3}+\frac{3}{2}\left(z^{2}-1\right) z \\
r^{3} Y_{3}^{1} & =-\frac{1}{48}(x+\mathbf{i} y)\left(360 z^{2}-72\right) \\
r^{3} Y_{3}^{2} & =15\left(x^{2}+2 \mathbf{i} x y-y^{2}\right) z \\
r^{3} Y_{3}^{3} & =-15 x^{3}+45 x y^{2}-15 \mathbf{i}\left(3 x^{2} y-y^{3}\right)
\end{aligned}
$$

$n=4$ :

$$
r^{4} Y_{4}^{0}=z^{4}+3\left(z^{2}-1\right) z^{2}+\frac{3}{8}\left(z^{2}-1\right)^{2}
$$




$$
\begin{aligned}
r^{4} Y_{4}^{1} & =-\frac{1}{384}(x+\mathbf{i} y)\left(3840 z^{3}+2880\left(z^{2}-1\right) z\right) \\
r^{4} Y_{4}^{2} & =\frac{1}{384}\left(x^{2}+2 \mathbf{i} x y-y^{2}\right)\left(20160 z^{2}-2880\right) \\
r^{4} Y_{4}^{3} & =-105\left(x^{3}-3 x y^{2}+\mathbf{i}\left(3 x^{2} y-y^{3}\right)\right) z \\
r^{4} Y_{4}^{4} & =105 x^{4}-630 x^{2} y^{2}+105 y^{4}+105 \mathbf{i}\left(4 x^{3} y-4 x y^{3}\right)
\end{aligned}
$$

$n=5$ :

$$
\begin{aligned}
r^{5} Y_{5}^{0} & =z^{5}+5\left(z^{2}-1\right) z^{3}+\frac{15}{8}\left(z^{2}-1\right)^{2} z \\
r^{5} Y_{5}^{1} & =-\frac{1}{3840}(x+\mathbf{i} y)\left(57600 z^{4}+86400\left(z^{2}-1\right) z^{2}+7200\left(z^{2}-1\right)^{2}\right) \\
r^{5} Y_{5}^{2} & =\frac{1}{3840}\left(x^{2}+2 \mathbf{i} x y-y^{2}\right)\left(403200 z^{3}+201600\left(z^{2}-1\right) z\right) \\
r^{5} Y_{5}^{3} & =-\frac{1}{3840}\left(x^{3}-3 x y^{2}+\mathbf{i}\left(3 x^{2} y-y^{3}\right)\right)\left(1814400 z^{2}-201600\right) \\
r^{5} Y_{5}^{4} & =945\left(x^{4}-6 x^{2} y^{2}+y^{4}+I\left(4 x^{3} y-4 x y^{3}\right)\right) z \\
r^{5} Y_{5}^{5} & =-945 x^{5}+9450 x^{3} y^{2}-4725 x y^{4}-945 \mathbf{i}\left(5 x^{4} y-10 x^{2} y^{3}+y^{5}\right)
\end{aligned}
$$

$n=6$ :

$$
\begin{aligned}
r^{6} Y_{6}^{0}= & z^{6}+\frac{15}{2}\left(z^{2}-1\right) z^{4}+\frac{45}{8}\left(z^{2}-1\right)^{2} z^{2}+\frac{5}{16}\left(z^{2}-1\right)^{3} \\
r^{6} Y_{6}^{1}= & -\frac{1}{46080}(x+\mathbf{i} y)\left(967680 z^{5}+2419200\left(z^{2}-1\right) z^{3}+604800\left(z^{2}-1\right)^{2} z\right) \\
r^{6} Y_{6}^{2}= & \frac{1}{46080}\left(x^{2}+2 \mathbf{i} x y-y^{2}\right) \\
& \left(9676800 z^{4}+9676800\left(z^{2}-1\right) z^{2}+604800\left(z^{2}-1\right)^{2}\right) \\
r^{6} Y_{6}^{3}= & -\frac{1}{46080} \\
& \left(x^{3}-3 x y^{2}+\mathbf{i}\left(3 x^{2} y-y^{3}\right)\right)\left(58060800 z^{3}+21772800\left(z^{2}-1\right) z\right) \\
r^{6} Y_{6}^{4}= & \frac{1}{46080} \\
& \left(x^{4}-6 x^{2} y^{2}+y^{4}+\mathbf{i}\left(4 x^{3} y-4 x y^{3}\right)\right)\left(239500800 z^{2}-21772800\right) \\
r^{6} Y_{6}^{5}= & -10395\left(x^{5}-10 x^{3} y^{2}+5 x y^{4}+\mathbf{i}\left(5 x^{4} y-10 x^{2} y^{3}+y^{5}\right)\right) z
\end{aligned}
$$




$$
\begin{aligned}
r^{6} Y_{6}^{6}= & 10395 x^{6}-155925 x^{4} y^{2}+155925 x^{2} y^{4}-10395 y^{6} \\
& +10395 \mathbf{i}\left(6 x^{5} y-20 x^{3} y^{3}+6 x y^{5}\right)
\end{aligned}
$$

